\title{
On an unconditionally convergent stabilized finite element approximation of resistive magnetohydrodynamics
}

\author{
Santiago Badia ${ }^{\mathrm{a}, \mathrm{b}}$, Ramon Codina ${ }^{\mathrm{b}}$, Ramon Planas ${ }^{\mathrm{a}}$ \\ ${ }^{a}$ Centre Internacional de Metodes Numerics a l'Enginyeria (CIMNE), \\ Parc Mediterrani de la Tecnologia, UPC \\ Esteve Terrades 5, 08860 Castelldefels, Spain. \\ sbadia@cimne.upc.edu, rplanas@cimne.upc.edu \\ ${ }^{b}$ Universitat Politecnica de Catalunya \\ Jordi Girona 1-3, Edifici C1, 08034 Barcelona, Spain. \\ ramon. codina@upc.edu
}

\begin{abstract}
In this work, we propose a new stabilized finite element formulation for the approximation of the resistive magnetohydrodynamics equations. The novelty of this formulation is the fact that it always converges to the physical solution, even for singular ones. A detailed set of numerical experiments have been performed in order to validate our approach.
\end{abstract}

Keywords: magnetohydrodynamics, finite elements, singular solutions, stabilized finite element methods

\section{Introduction}

\subsection{State-of-the-art}

In this work, we propose a novel numerical formulation for the approximation of the incompressible visco-resistive magnetohydrodynamics (MHD) system that models incompressible viscous and electrically conducting fluids under the influence of electromagnetic fields (see [22]). Examples of such fluids include liquid metals and plasmas. The numerical approximation of the associated set of partial differential equations (PDEs) is of paramount importance in fusion energy, since it allows us to model liquid metal cooling and He-Tr extraction systems in fusion reactors (the so-called breeding blankets) as well as the plasma confinement in the core [33].

Many conforming numerical approximations to this problem have been proposed so far. There are different equivalent formulations of the continuous magnetic sub-problem, namely saddle-point and (weighted) exact penalty formulations (see [40] and [27, 1, 28, 19, 21] respectively). The first one leads to a double-saddle-point formulation for the MHD system. A Galerkin finite element (FE) approximation of the resulting problem has been proposed and analyzed by Schötzau in [40]. It is well-known that saddle-point formulations require to choose particular mixed FE spaces satisfying 
discrete versions of the so-called inf-sup conditions (see e.g. [8]). It complicates the implementation issues, e.g. the database structure, the computation of the coupling terms and the graphs needed for the compressed storage of the system matrix (see Section 1.2). Instead, a weighted exact penalty formulation has been used in [27]. This formulation allows to simplify the aforementioned implementation issues but introduces a new complication, the definition of the weight function (see [21]); it requires an a priori knowledge of the exact solution -where the singularities are placed- and the final expressions are hard to integrate accurately using numerical integration. Since the resistive MHD system loses coercivity as the Reynolds and magnetic Reynolds numbers increase, i.e. convectiontype terms become dominant, a mixed FE formulation of the problem is unstable unless the mesh size is sufficientely refined, which is impractical.

Alternative formulations have been proposed for a regularized version of the system, based on an exact penalty formulation. ${ }^{1}$ Under some assumptions on the computational domain $\Omega$, the magnetic field is smoother than for the original problem (see e.g. [25]). An inf-sup stable Galerkin FE formulation for the flow sub-problem and the regularized exact penalty formulation for the magnetic sub-problem has been proposed in [26], whereas a stabilized FE version of this formulation can be found in [24].

Non-conforming approximations of discontinuous Galerkin type have been designed in [29]. These methods have good numerical properties, but the increase in CPU cost-degrees of freedom- of these formulations (with respect to conforming formulations) is severe for realistic large-scale applications.

\subsection{Motivation of the work}

The multi-physics nature of the MHD system, and the fact that both sub-problems have a saddle-point structure (before modifications), makes the numerical approximation of this PDE system a challenging task. Further, the extremely complex nature of the phenomena that can be modeled [11, 12], requires the development of low-storage algorithms that allow an easy-to-optimize implementation suitable for massive parallelization.

A straightforward Galerkin FE approximation of the original problem would require very specific FE spaces for the different unknowns in order to satisfy the corresponding inf-sup conditions, e.g. the MINI element could be used for the fluid sub-problem whereas weakly-solenoidal Nédélec bases are needed for the magnetic sub-problem [36, 37]. Clearly, this approach introduces implementational complications in comparison to a straightforward equal interpolation of all the vectorial components and scalar unknowns, e.g. database structures or integration of coupling terms. More important, for $\mathrm{n}_{\mathrm{unk}}$ unknowns, ${ }^{2}$ we require (in general) $\left(\mathrm{n}_{\mathrm{unk}}\right)^{2}$ graphs to define the sparse structures of the $\left(\mathrm{n}_{\mathrm{unk}}\right)^{2}$ block matrices. The optimization of a Galerkin-type MHD implementation is a formidable task. For these reasons, the use of stabilized FE formulations that allow equal interpolation for all the unknowns becomes a very appealing discretization technique for multi-physics applications (see [4]). This way, with only one graph -the mesh-graph- we are able to define the sparse structure of the matrix.

\footnotetext{
${ }^{1}$ All these methods must be used with caution, since they converge to spurious solutions when the exact magnetic field is not smooth.

${ }^{2}$ The MHD system at hand involves fluid velocity and pressure as well as magnetic field for the penalty formulation $\left(\mathrm{n}_{\mathrm{unk}}=3\right)$ and an additional magnetic pseudo-pressure for the double-saddle-point structure $\left(\mathrm{n}_{\mathrm{unk}}=4\right)$. Herein we use the concept of magnetic pseudo-pressure to denote the Lagrange multiplier that is introduced to enforce the solenoidal constraint on the magnetic field. Do not confuse it with the classical notion of magnetic pressure in MHD (see [22]).
} 
However, implementational issues are not the only (or even the main) justification to use stabilized formulations instead of mixed ones. The additional stabilization terms not only avoid the fulfillment of the inf-sup conditions but add streamline-diffusion to reduce oscillations in dominant convection flows [9]. ${ }^{3}$ Furthermore, the introduction of stabilization terms changes the nature of the problem, and subsequently the system matrix. Whereas the system matrix obtained by using mixed FEs is indefinite, with null diagonal-blocks, the one from the stabilized FE formulation is positive definite. It simplifies the numerical linear algebra strategy to solve the final linear system in an efficient (optimal) way (see [41] for a detailed discussion).

With regard to parallelization, the possibility to work with the mesh-graph only simplifies the required graph-partitioning needed in domain decomposition approaches [45]. Further, the ease in the definition of effective preconditioners also helps to attain algorithmic scalability [41].

Some stabilized FE formulations have been proposed so far for resistive MHD [6, 7, 24, 25, 16, 17 , 41]. These formulations share the benefits listed above but they are based on the regular functional setting of the problem, and so, restricted to smooth or convex domains (see [21]). They are accurate for regular magnetic solutions but tend to spurious (unphysical) solutions otherwise (see Section 3 for further discussion). The objective of this work is to propose a novel stabilized FE formulation that always converges to the exact (physical) solution.

More specifically, the objective of this work is to design and analyze a numerical FE formulation of the MHD problem with the following features:

- always converges to the physical solution, even when it is a singular solution,

- allows equal Lagrangian interpolation of every component of the vectorial fields as well as the scalar fields, ${ }^{4}$

- allows for arbitrary-order interpolation,

- introduces effective numerical stability in convection-dominated regimes,

- does not require any a priori information of the solution, i.e. it is a fully automatic approach,

always keeping optimal a priori error estimates for smooth solutions.

The outline of this work is the following. First, the MHD problem of interest is stated in Section 2. Some existing FE discretizations (in space) are detailed in Section 3 as well as some notation. The linearization and time integration of the resulting problem is presented in Section 4. Section 5 is devoted to the formulation we propose. A complete set of numerical experiments that validate the formulation and are in concordance with the numerical analyses in [5] are presented in Section 6. We finish the work by drawing some conclusions in Section 7.

\footnotetext{
${ }^{3}$ Most of the applications of interest are in this regime.

${ }^{4}$ This feature implies that only one graph is enough for the definition of the sparse structure of the system matrix.
} 


\section{Problem statement}

\subsection{The strong form}

The incompressible visco-resistive MHD system of partial differential equations consists of the NavierStokes equations coupled to the (simplified) Maxwell equations via the Lorentz force. It reads as follows: find a velocity field $\mathbf{u}(\mathbf{x}, t)$, a (dynamic) pressure $\tilde{p}(\mathbf{x}, t)$ and an induced magnetic field $\mathbf{b}(\mathbf{x}, t)$ such that

$$
\begin{aligned}
\rho \partial_{t} \mathbf{u}+\rho \mathbf{u} \cdot \nabla \mathbf{u}-\mu_{\mathrm{f}} \Delta \mathbf{u}+\nabla \tilde{p}-\frac{1}{\mu_{\mathrm{m}}}(\nabla \times \mathbf{b}) \times \mathbf{b} & =\mathbf{f}_{\mathbf{u}}, \\
\nabla \cdot \mathbf{u} & =0, \\
\partial_{t} \mathbf{b}+\frac{1}{\mu_{\mathrm{m}}} \nabla \times\left(\frac{1}{\sigma} \nabla \times \mathbf{b}\right)-\nabla \times(\mathbf{u} \times \mathbf{b}) & =\mathbf{f}_{\mathrm{b}}, \\
\nabla \cdot \mathbf{b} & =0,
\end{aligned}
$$

in $(\mathbf{x}, t) \in \Omega \times(0, T)$, where $\partial_{t}$ stands for the partial time derivative $\left(\mathrm{d}_{t}\right.$ will be used for the total one), $\Omega \subset \mathbb{R}^{d}$ is the spatial open bounded domain filled by the fluid (assumed polyhedral in the finite element approximation), $d$ being the space dimension, and $(0, T)$ is the time interval of interest. $\mathbf{f}_{\mathrm{u}}$ and $\mathbf{f}_{\mathrm{b}}$ are the forcing terms, $\mathbf{f}_{\mathrm{b}}$ being solenoidal. With regard to the physical parameters that describe the fluid, $\rho$ is its density, $\mu_{\mathrm{f}}$ the fluid viscosity, $\mu_{\mathrm{m}}$ the magnetic permeability and $\sigma$ the electric conductivity. In this work, we consider all physical properties constant. These equations must be supplemented with appropriate boundary and initial conditions. The initial conditions are:

$$
\mathbf{u}(\mathbf{x}, 0)=\mathbf{u}_{0}(\mathbf{x}), \quad \mathbf{b}(\mathbf{x}, 0)=\mathbf{b}_{0}(\mathbf{x}),
$$

where $\mathbf{b}_{0}$ must be solenoidal. This restriction can be weakened for $\mathbf{u}_{0}$, but it introduces a singularity at $t=0$ (see [44]).

We can easily check that this system is over-constrained, since no Lagrange multiplier has been used to enforce the null-divergence restriction over the magnetic field $\mathbf{b}$. However, the problem has at least one solution; taking the divergence of $(1 \mathrm{c})$, we obtain $\partial_{t}(\nabla \cdot \mathbf{b})=0$. This fact, together with the solenoidal initial condition, amounts to say that (1d) is satisfied at all times. Unfortunately, this procedure cannot be used for the discretized system when Lagrangian finite elements are used. In this case, it is more suitable to explicitly enforce (1d) via a Lagrange multiplier, the magnetic pseudopressure $r(\mathbf{x}, t)$. Let us introduce the following augmented formulation of (1), re-scaled in a more suitable way for the subsequent exposition:

$$
\begin{aligned}
\partial_{t} \mathbf{u}+\mathbf{u} \cdot \nabla \mathbf{u}-\nu \Delta \mathbf{u}+\nabla p-(\nabla \times \mathbf{b}) \times \varrho \mathbf{b} & =\mathbf{f}_{\mathbf{u}}, \\
\nabla \cdot \mathbf{u} & =0, \\
\varrho \partial_{t} \mathbf{b}+\lambda \nabla \times(\nabla \times \mathbf{b})+\nabla r-\nabla \times(\mathbf{u} \times \varrho \mathbf{b}) & =\mathbf{f}_{\mathrm{b}}, \\
\nabla \cdot \mathbf{b} & =0,
\end{aligned}
$$

where $\nu:=\mu_{\mathrm{f}} \rho^{-1}, \varrho:=\left(\rho \mu_{\mathrm{m}}\right)^{-1}$ and $\lambda:=\left(\rho \mu_{\mathrm{m}}^{2} \sigma\right)^{-1} ; p:=\rho^{-1} \tilde{p}$ is the kinematic pressure. $\mathbf{f}_{\mathrm{u}}$ and $\mathbf{f}_{\mathrm{b}}$ have been re-defined accordingly.

In order to introduce the boundary conditions, let us consider two disjoint partitions of the domain boundary $\Gamma \equiv \partial \Omega$ :

$$
\Gamma=\Gamma_{\mathrm{f}, \mathrm{e}} \cup \Gamma_{\mathrm{f}, \mathrm{n}}, \quad \Gamma=\Gamma_{\mathrm{m}, \mathrm{e}} \cup \Gamma_{\mathrm{m}, \mathrm{n}},
$$


where the first subscript denotes the subproblem (f for fluid and $m$ for magnetic) and the second one the type of boundary condition (e for essential and $n$ for natural). Then, the fluid sub-problem is supplemented with the standard boundary conditions:

$$
\mathbf{u}=\mathbf{u}_{\Gamma} \quad \text { on } \Gamma_{\mathrm{f}, \mathrm{e}}, \quad-p \mathbf{n}+\nu \mathbf{n} \cdot \nabla \mathbf{u}=\boldsymbol{\sigma}_{n, \Gamma} \quad \text { on } \Gamma_{\mathrm{f}, \mathrm{n}},
$$

where $\mathbf{u}_{\Gamma}(\mathbf{x}, t)$ and $\boldsymbol{\sigma}_{n, \Gamma}(\mathbf{x}, t)$ are the trace and normal stress prescribed; $\mathbf{n}(\mathbf{x})$ denotes the normal vector on $\Gamma$ pointing outwards from $\Omega$. With regard to the magnetic sub-problem, we consider the set of ideal boundary conditions:

$$
\mathbf{n} \times \mathbf{b}=\mathbf{n} \times \mathbf{b}_{\Gamma}, \quad r=0 \quad \text { on } \Gamma_{\mathrm{m}, \mathrm{e}}, \quad \mathbf{n} \cdot \mathbf{b}=\mathbf{n} \cdot \mathbf{b}_{\Gamma}, \quad \mathbf{n} \times(\nabla \times \mathbf{b})=\mathbf{J}_{\Gamma} \quad \text { on } \Gamma_{\mathrm{m}, \mathrm{n}},
$$

where $\mathbf{n} \cdot \mathbf{b}_{\Gamma}$ and $\mathbf{n} \times \mathbf{b}_{\Gamma}$ are the normal and tangential traces to be prescribed; clearly, $\mathbf{J}_{\Gamma} \cdot \mathbf{n}$ must vanish.

\subsection{The weak form}

\subsubsection{Notation}

Let us introduce some notation to set up the weak form of the problem. As usual, Sobolev spaces of functions whose derivatives of order up to $m$ belong to $L^{2}(\Omega)$ are denoted by $H^{m}(\Omega) ; H_{0}^{1}(\Omega)$ is the subspace of $H^{1}(\Omega)$ of functions vanishing on $\partial \Omega$. The space of vector functions with components in $L^{2}(\Omega)$ and with divergence also in $L^{2}(\Omega)$ is denoted $H(\operatorname{div} ; \Omega)$; if the components are $L^{2}(\Omega)$ and the curl is in $L^{2}(\Omega)^{d}$ the space is denoted $H(\operatorname{curl} ; \Omega) . H(\operatorname{div} 0 ; \Omega)$ is the subspace of $L^{2}(\Omega)^{d}$ of divergence free vector functions.

The inner product of $f, g \in L^{2}(\Omega)$ is represented as $(f, g)$, whereas $\langle f, g\rangle$ is used to denote the integral $\int_{\Omega} f g$ whenever it makes sense; this in particular applies for the duality between $H_{0}^{1}(\Omega)$ and its topological dual $H^{-1}(\Omega)$. The same notation is used for both scalar and vector valued functions.

Given a normed functional space $X$, its norm is written as $\|\cdot\|_{X}$, with the abbreviations $\|\cdot\|_{L^{2}(\Omega)} \equiv$ $\|\cdot\|,\|\cdot\|_{H^{m}(\Omega)} \equiv\|\cdot\|_{m},\|\cdot\|_{H^{-1}(\Omega)} \equiv\|\cdot\|_{-1},\|\cdot\|_{H(\text { curl } ; \Omega)} \equiv\|\cdot\|_{\text {curl }}$. Functions defined on the time interval $(0, T)$ whose $X$-norm is in $L^{2}(0, T)$ are denoted by $L^{2}(0, T ; X)$, whereas distributions in $(0, T)$ whose $X$-norm is bounded are represented by $\mathcal{D}^{\prime}(0, T ; X)$.

Finally, the symbol $\lesssim$ is used to denote $\leq$ up to positive constants.

\subsubsection{A double saddle-point formulation}

Let us consider the functional setting in which the system of equations (2) is well-posed. For the sake of clarity, we will consider homogeneous essential boundary conditions; in any case, the extension to the most general case is standard. We introduce the vectorial functional spaces:

$$
\begin{aligned}
& \boldsymbol{V}=\left\{\mathbf{v} \in H^{1}(\Omega)^{d} \text { such that } \mathbf{v}=\mathbf{0} \text { on } \Gamma\right\}, \\
& \boldsymbol{C}=\{\mathbf{c} \in H(\mathbf{c u r l} ; \Omega) \text { such that } \mathbf{n} \times \mathbf{c}=\mathbf{0} \text { on } \Gamma\},
\end{aligned}
$$

for the velocity and magnetic field functions, respectively. Further, the space for fluid pressures is $Q \equiv L_{0}^{2}(\Omega)$ and the one for magnetic pseudo-pressures $S \equiv H_{0}^{1}(\Omega)$. Now, we can state the 
transient MHD problem at hand in its weak form as follows: find $\mathbf{u} \in L^{2}(0, T ; \boldsymbol{V}), \mathbf{b} \in L^{2}(0, T ; \boldsymbol{C})$, $p \in \mathcal{D}^{\prime}(0, T ; Q)$ and $r \in \mathcal{D}^{\prime}(0, T ; S)$ such that

$$
\begin{aligned}
\mathrm{d}_{t}(\mathbf{u}, \mathbf{v})+\langle\mathbf{u} \cdot \nabla \mathbf{u}, \mathbf{v}\rangle+(\nu \nabla \mathbf{u}, \nabla \mathbf{v})-(p, \nabla \cdot \mathbf{v})-\langle(\nabla \times \mathbf{b}) \times \varrho \mathbf{b}, \mathbf{v}\rangle & =\left\langle\mathbf{f}_{\mathrm{u}}, \mathbf{v}\right\rangle, \\
(q, \nabla \cdot \mathbf{u}) & =0, \\
\mathrm{~d}_{t}(\varrho \mathbf{b}, \mathbf{c})+(\lambda \nabla \times \mathbf{b}, \nabla \times \mathbf{c})-\langle\nabla \times(\mathbf{u} \times \varrho \mathbf{b}), \mathbf{c}\rangle+(\nabla r, \mathbf{c}) & =\left\langle\mathbf{f}_{\mathrm{b}}, \mathbf{c}\right\rangle, \\
-(\nabla s, \mathbf{b}) & =0,
\end{aligned}
$$

for any $(\mathbf{v}, \mathbf{c}, q, s) \in(\boldsymbol{V}, \boldsymbol{C}, Q, S)$ almost everywhere (a.e.) in $(0, T)$. Let us show that $r \equiv 0$ in (4). Taking $\mathbf{c}=\nabla r$ (which clearly belongs to $C$ ) in (4c), and using the fact that $\nabla \times \nabla r=\mathbf{0}$ and $\nabla \cdot \mathbf{f}_{\mathrm{b}}=0$ a.e. in $\Omega$, we obtain $\|\nabla r\|=0$. Since $r$ vanishes on $\partial \Omega$, it implies $r \equiv 0$ a.e. in $\Omega$ by virtue of Poincaré's inequality. We refer to [28, Propositions 3.4 and 3.5] for the completion of the proof.

Let us re-write system (4) in compact manner as:

$\mathcal{M}((\mathbf{u}, \mathbf{b}),(\mathbf{v}, \mathbf{c}))+\mathcal{A}((\mathbf{u}, \mathbf{b}, p, r),(\mathbf{v}, \mathbf{c}, q, s))=\left\langle\mathbf{f}_{\mathbf{u}}, \mathbf{v}\right\rangle+\left\langle\mathbf{f}_{\mathbf{b}}, \mathbf{c}\right\rangle, \quad \forall(\mathbf{v}, \mathbf{c}, q, s) \in \boldsymbol{V} \times \boldsymbol{C} \times Q \times S$,

where the form $\mathcal{M}(\cdot)$ includes all the time derivative terms and $\mathcal{A}(\cdot)$ the rest of left-hand side terms.

The problem is well-posed under the assumption of small data, due to the inf-sup conditions

$$
\inf _{q \in Q} \sup _{\mathbf{v} \in \boldsymbol{V}} \frac{(q, \nabla \cdot \mathbf{v})}{\|q\|\|\mathbf{v}\|_{1}} \geq \beta_{\mathrm{f}}>0, \quad \inf _{s \in S} \sup _{\mathbf{c} \in \boldsymbol{C}} \frac{(\nabla s, \mathbf{c})}{\|s\|_{1}\|\mathbf{c}\|_{H(\mathbf{c u r l} ; \Omega)}} \geq \beta_{\mathrm{m}}>0
$$

that are known to be true at the continuous level, as well as the Poincaré-Friedrichs inequalities

$$
\begin{aligned}
\|\mathbf{v}\|_{1} & \leq C_{\mathrm{P}, 1}\|\nabla \mathbf{v}\| & & \text { for } \mathbf{v} \in H_{0}^{1}(\Omega)^{d}, \\
\|\mathbf{c}\|_{H(\mathbf{c u r l} ; \Omega)} & \leq C_{\mathrm{P}, 2}\|\nabla \times \mathbf{c}\| & & \text { for } \mathbf{c} \in \boldsymbol{C} \cap H(\operatorname{div} 0 ; \Omega)
\end{aligned}
$$

(see [35, Corollary 3.51]). We refer to $[39,40]$ for a mathematical discussion about the well-posedness of this problem.

\subsubsection{A (weighted) exact penalty formulation for the magnetic sub-problem}

As we will discuss later on, the numerical approximation of saddle-point PDEs is complicated. So, we can consider an alternative formulation for the magnetic sub-problem, in which Eqs. (4c)-(4d) are replaced by

$$
\mathrm{d}_{t}(\varrho \mathbf{b}, \mathbf{c})+(\lambda \nabla \times \mathbf{b}, \nabla \times \mathbf{c})-\langle\nabla \times(\mathbf{u} \times \varrho \mathbf{b}), \mathbf{c}\rangle+\lambda(\nabla \cdot \mathbf{b}, \nabla \cdot \mathbf{c})=\left\langle\mathbf{f}_{\mathrm{b}}, \mathbf{c}\right\rangle .
$$

We can easily check that systems (4) and (4a)-(4b)-(7) are equivalent (see [28, 20]). This way, the only inf-sup condition that is needed in order for the problem to be well-posed is the one related to the fluid sub-problem. This formulation can be understood as an exact penalty strategy. Some numerical approximations of the MHD system have considered this statement of the problem [26, 24]. Let us remark that in this work, for the reasons commented below, the numerical methods that are proposed approximate the double-saddle point formulation (4). 


\begin{tabular}{|c|c|c|c|c|c|c|}
\hline \multirow[t]{2}{*}{ Fluid sub-problem } & \multicolumn{3}{|c|}{ Magnetic sub-problem (regularized) } & \multicolumn{3}{|c|}{ Magnetic sub-problem (original) } \\
\hline & $\begin{array}{l}\text { Inf-sup } \\
\text { stable }\end{array}$ & $\begin{array}{l}\text { Exact } \\
\text { penalty }\end{array}$ & $\begin{array}{l}\text { Stabilized } \\
\text { method }\end{array}$ & $\begin{array}{l}\text { Inf-sup } \\
\text { stable }\end{array}$ & $\begin{array}{l}\text { Weighted } \\
\text { penalty }\end{array}$ & $\begin{array}{l}\text { Stabilized } \\
\text { method }\end{array}$ \\
\hline $\begin{array}{l}\text { Inf-sup } \\
\text { stable }\end{array}$ & & $\begin{array}{l}\text { Gunzburger } \\
\text { et al., [26] }\end{array}$ & & $\begin{array}{l}\text { Schötzau, } \\
{[40]}\end{array}$ & $\begin{array}{l}\text { Hasler et } \\
\text { al., [27] }\end{array}$ & \\
\hline $\begin{array}{l}\text { Stabilized } \\
\text { method }\end{array}$ & & $\begin{array}{l}\text { Gerbeau, } \\
{[24]}\end{array}$ & $\begin{array}{l}\text { Codina et } \\
\text { al., [16] }\end{array}$ & & & This work \\
\hline
\end{tabular}

Table 1: References for different combinations of fluid and magnetic techniques for the MHD problem. For the magnetic sub-problem, we have distinguished among the methods that use the original functional setting and the ones that use the regularized setting (not suitable for singular solutions).

\section{Some finite element approximations}

In this section, we show some of the different alternatives proposed so far for the numerical approximation of the resistive MHD system (1) using finite element techniques. In some cases, the references that are provided considered the stationary version of the problem, but it does not affect the forthcoming discussion. First, we list FE approximations that are based on the different continuous formulations of the MHD problem. We can distinguish among the approximations that are suitable for singular solutions of the magnetic field, i.e. they are posed in the original functional setting $H(\operatorname{curl} ; \Omega) \times H^{1}(\Omega)$, and those that use the regularized formulation, only suitable for convex or smooth enough domains. In this case $\mathbf{b} \in H^{1}(\Omega)^{d}$. Table 1 shows different combinations of fluid and magnetic sub-problem numerical approximations. We distinguish among methods able to capture singular magnetic solutions and regularized ones. Further, we indicate the references in which every combination has been proposed. All those methods are explained below.

Let us start with formulation suitable for singular magnetic solutions. Since the problem at hand couples two saddle-point sub-problems, a Galerkin FE approximation should satisfy a discrete version of the two inf-sup conditions (5) above. In order to do this, we rely on the well-established mixed FE theory (see e.g. [8]). Since the two inf-sup conditions are different, stable approximations for the $(\mathbf{u}, p)$ pair differ from those stable for the magnetic pair $(\mathbf{b}, r)$. E.g. the fluid sub-problem could be solved by using the MINI element, whereas the magnetic problem should be approximated by a Nédélec FE space (see [36, 37]). The use of inf-sup stable elements for every sub-problem is the strategy used in [40]. From a theoretical point of view, this is a satisfactory approach, because the final MHD solver is able to approximate singular solutions, with $\mathbf{b} \notin H^{1}(\Omega)^{d}$. However, this approach is not appealing from a practical point of view, as commented in Section 1.2.

A way to simplify the numerical approximation consists in the use of an exact penalty formulation for the magnetic field. Under the following assumption over the domain:

$$
\text { The domain } \Omega \text { is convex or its boundary is } \mathcal{C}^{1,1}
$$

we can prove that the magnetic field does belong to $H^{1}(\Omega)^{d}$ (see [25]). This approach was proposed in [26] in the Galerkin case. In [24], a stabilized FE formulation for the fluid sub-problem as well as a Galerkin FE discretization of the exact penalty formulation for the magnetic sub-problem are proposed. The method allows one-graph implementation, arbitrary-order Lagrangian interpolations for all the unknowns (velocity, pressure and magnetic field), and stabilizes convection. However, the 
straightforward exact penalty formulation only provides magnetic fields in $H^{1}(\Omega)^{d}[20,3]$. Further, $H^{1}(\Omega)^{d}$ is a strict closed subspace of $H(\operatorname{curl} ; \Omega) \cap H(\operatorname{div} ; \Omega)$ and so, $H^{1}$-conforming approximations do not have an approximability property (see e.g. [23]). As a results, the formulations based on the exact penalty lead to spurious numerical solutions in general. The formulation proposed in [17] uses a regularized double saddle-point formulation of the problem at hand, in order to explicitly enforce the free-divergence constraint over the magnetic field. Stabilized FE discretizations of both the fluid and magnetic sub-problem are considered. It shares the same benefits and limitations of the previous approach. From the conceptual point of view, the formulation is motivated from the variational multiscale framework (see $[30,31]$ ) and the stabilization parameters are carefully designed from the convergence analysis. Both aspects are shared by the formulation we propose in Section 5.

In order to solve this situation, less control over $\mathbf{b}$ is required, e.g. with a weighting in the penalty term. Costabel and Dauge proposed in [21] to replace the penalty term in (7) by a weighted one (in the frame of the electrostatic Maxwell problem) of the form $(\omega \lambda \nabla \cdot \mathbf{b}, \nabla \cdot \mathbf{c})$ where $\omega(\mathbf{x})$ is the weight function, that can only be effectively defined knowing a priori the location of the singularities (see [20]). It has been proved in [20] that a proper choice of this weighting allows one to capture singular solutions. The weighted exact penalty together with an inf-sup stable Galerkin approximation of the fluid problem has been proposed in [27] for the MHD problem (1).

\section{Time discretization and linearization}

All the developments in this work are for the spatial discretization of the MHD system (4). The stabilized FE formulation we propose can be applied to any time integration scheme. In particular, we can consider $\theta$-methods for the time discretization. After spatial discretization, our time-continuous formulation can be written as a differential-algebraic equation:

$$
\dot{\boldsymbol{x}}(t)=A(\boldsymbol{x}(t), \boldsymbol{y}(t), t), \quad \mathbf{0}=C(\boldsymbol{x}(t), \boldsymbol{y}(t), t),
$$

where $\boldsymbol{x}(t) \in \mathbb{R}^{n}, \boldsymbol{y}(t) \in \mathbb{R}^{m}, A: \mathbb{R}^{n+m} \rightarrow \mathbb{R}^{n}$ and $C: \mathbb{R}^{n+m} \rightarrow \mathbb{R}^{m}$. Using the definitions

$$
\dot{\boldsymbol{x}}^{n+1}=\frac{\boldsymbol{x}^{n+1}-\boldsymbol{x}^{n}}{\delta t}, \quad \boldsymbol{x}^{n+\theta}=\theta \boldsymbol{x}^{n+1}+(1-\theta) \boldsymbol{x}^{n},
$$

(analogously for $\boldsymbol{y}^{n+\theta}$ ), the time discretization of this problem with the $\theta$-method simply reads as:

$$
\dot{\boldsymbol{x}}^{n+1}=A\left(\boldsymbol{x}^{n+\theta}, \boldsymbol{y}^{n+\theta}, t^{n+\theta}\right), \quad \mathbf{0}=C\left(\boldsymbol{x}^{n+\theta}, \boldsymbol{y}^{n+\theta}, t^{n+\theta}\right), \quad \text { for } \theta \in(0,1] .
$$

We consider the following time-discretization of (2):

$$
\begin{aligned}
\dot{\mathbf{u}}^{n+1}+\hat{\mathbf{u}}^{n} \cdot \nabla \mathbf{u}^{n+\theta}-\nu \Delta \mathbf{u}^{n+\theta}+\nabla p^{n+\theta}-\left(\nabla \times \mathbf{b}^{n+\theta}\right) \times \varrho \hat{\mathbf{b}}^{n} & =\mathbf{f}_{\mathbf{u}}, \\
\nabla \cdot \mathbf{u}^{n+\theta} & =0, \\
\varrho \dot{\mathbf{b}}^{n+1}+\lambda \nabla \times\left(\nabla \times \mathbf{b}^{n+\theta}\right)+\nabla r^{n+\theta}-\nabla \times\left(\mathbf{u}^{n+\theta} \times \varrho \hat{\mathbf{b}}^{n}\right) & =\mathbf{f}_{\mathrm{b}}, \\
\nabla \cdot \mathbf{b}^{n+\theta} & =0,
\end{aligned}
$$

where $\hat{\mathbf{u}}^{n}$ and $\hat{\mathbf{b}}^{n}$ can be either time extrapolations to approximate $\mathbf{u}^{n+\theta}$ and $\mathbf{b}^{n+\theta}$, respectively, or previous iterates within a nonlinear iteration strategy to compute the unknowns at time level $n+1$. 
In the first case, we may choose $\hat{\mathbf{u}}^{n}=\mathbf{u}^{n}, \hat{\mathbf{b}}^{n}=\mathbf{b}^{n}$ if $\theta \neq 1 / 2$ (first order schemes) or $\hat{\mathbf{u}}^{n}=$ $2 \mathbf{u}^{n}-\mathbf{u}^{n-1}, \hat{\mathbf{b}}^{n}=2 \mathbf{b}^{n}-\mathbf{b}^{n-1}$ if $\theta=1 / 2$ (second order scheme). In the second case, the linearization can be considered of Picard's type. In fact, it can be easily checked that this is the only fixed-point linearization of the problem that leads to an absolutely stable algorithm in time (see [17]). Segregated time-marching schemes that decouple the sub-problems are conditionally stable and not suitable when the coupling is important. For the sake of conciseness, we will consider $\hat{\mathbf{u}}^{n}=\mathbf{u}^{n}, \hat{\mathbf{b}}^{n}=\mathbf{b}^{n}$ in the following.

\section{A stabilized FE formulation suitable for singular magnetic solutions}

Let us present now the spatial discretization we propose. Let $\mathcal{T}_{h}=\{K\}$ be a FE partition of the domain $\Omega$. For simplicity we assume $\Omega$ polyhedral and $\mathcal{T}_{h}$ quasi-uniform, of diameter $h$. Summation over all the element domains $K$ is denoted as $\sum_{K}$. Finite element spaces and FE functions are identified with the subscript $h$. Only conforming approximations are considered, i.e., the FE spaces where the unknowns are sought are $\boldsymbol{V}_{h} \subset \boldsymbol{V}, \boldsymbol{C}_{h} \subset \boldsymbol{C}, Q_{h} \subset Q$ and $S_{h} \subset S$. In particular, in the applications we will be interested in the use of $\mathcal{C}^{0}$ Lagrangian finite element interpolations of an arbitrary order for all the unknowns. Given two functions $f$ and $g$ piecewise polynomial on each $K \in \mathcal{T}_{h}$, we define $(f, g)_{h}:=\sum_{K} \int_{K} f g$ and $\|f\|_{h}:=(f, f)_{h}^{1 / 2}$.

Since we assume $\mathcal{T}_{h}$ quasi-uniform, the following inverse inequality holds:

$$
\left\|\nabla v_{h}\right\|_{L^{2}(K)} \leq \frac{C_{\text {inv }}}{h}\left\|v_{h}\right\|_{L^{2}(K)}, \quad K \in \mathcal{T}_{h},
$$

for a positive constant $C_{\mathrm{inv}}$ and for all piecewise polynomial functions $v_{h}$.

We consider a residual-based stabilized FE formulation for the MHD problem. This type of formulation does not change the statement of the continuous problem but modifies the way the discretization is performed. Instead of considering only those terms that come from a Galerkin discretization, this type of formulation includes additional terms, that are always proportional to some residual, and so, consistent. In order for this approach to be effective, the new terms must provide stability over the Lagrange multiplier-type unknowns, in order to avoid the fulfillment of discrete inf-sup conditions, as well as convection stability (see e.g. [15]). These methods can be motivated as multiscale methods in which the sub-grid component of the solution is properly modeled. Different closures for the sub-grid component have been proposed so far; we can distinguish among standard variational multiscale formulations (also called algebraic sub-grid scales) and orthogonal sub-grid scales, enforced to be orthogonal to the FE functions in $L^{2}$ sense. Analogously, we can distinguish among standard quasi-static formulations, in which the time derivative of the sub-grid component is neglected, and dynamic closures; in this last case, the model for the sub-grid scale is an ordinary differential equation (see [13]). It is not the aim of this work to motivate the stabilized FE formulation we propose. The heuristic motivation of these methods can be found in any of the references above. Since we are presenting a novel formulation, we have considered the most standard type of formulation, an algebraic quasi-static closure. ${ }^{5}$

\footnotetext{
${ }^{5}$ Roughly speaking, the type of formulation we propose reads as follows. Given a PDE (in strong form) that reads as
} 
In order to obtain a numerical algorithm suitable for singular solutions and avoiding the need to define weighting functions that require information about the placement of singularities, we stick to the double saddle-point formulation (4).

This way, using the approach indicated above, the stabilized FE formulation we propose is stated in Algorithm 1.

Let us stress the key features of our formulation, that allow us to design an unconditionally convergent stabilized method with the interesting properties listed above. The stabilization terms in the NavierStokes equations are standard, as well as the definition of the corresponding stabilization parameters $\tau_{1}$ and $\tau_{2}$, apart from the coupling with the magnetic field; see [17] for a detailed justification. With regard to the magnetic equation, we have split its residual into two parts $\mathbf{R}_{\mathrm{b}, 1}$ and $\mathbf{R}_{\mathrm{b}, 2}$; these two terms are still residuals, since $r=0$. Further, we have included the solenoidal constraint residual $R_{r}$. Now, we are in position to pre-multiply these three residuals with different stabilization parameters: $\tau_{3}, \tau_{4}$ and $\tau_{5}$ respectively. On one hand, $\tau_{4} \sim \mathcal{O}(1)$ in order to have $H^{1}$-stability over $r_{h}$. On the other hand, $\tau_{3} \sim \mathcal{O}\left(h^{2}\right)$, in order for $\left(\nabla \times \nabla \times \mathbf{c}_{h}, \tau_{3} \nabla \times \nabla \times \mathbf{b}_{h}\right)_{h}$ to have sense for $\mathbf{b}_{h} \in H(\mathbf{c u r l} ; \Omega)$ only; we refer to [5] for the complete numerical analysis of the method. Even though it is not so obvious, we have to pick $\tau_{5} \sim \mathcal{O}\left(h^{2}\right)$ in order to have full stability of $\mathbf{b}_{h}$ in $H$ (curl; $\Omega$ ). We refer to [3] for a detailed justification of this fact via the numerical analysis for the Maxwell operator. ${ }^{6}$ Note that the stabilization parameters in Algorithm $1, \tau_{i}(i=1, \ldots, 5)$, depend on algorithmic constants $c_{j}$ $(j=1, \ldots, 7)$ as well as on a length scale $L_{0}$, and the asymptotic behavior just described assumes that these are fixed. This is common to all stabilized finite element methods, and thus it is not our purpose to discuss how to choose the constants. Results may be sensitive to their value for a fixed mesh, but the asymtotic behavior of the numerical formulation is independent of it, provided they are $\mathcal{O}(1)$. We indicate in the numerical examples how the constants have been taken.

At this point, let us comment the main difference among the proposed formulation (Algorithm 1) and the one in [17]. In this reference, the residual was not split, i.e. the gradient of the magnetic pseudo-pressure was included in the residual for $\mathbf{b}$ and $\tau_{3}=\tau_{4}$. Even though this might seem the most natural option, the convergence analysis leads to the need to take the stabilization parameters as $\tau_{3}=\tau_{4} \sim \mathcal{O}\left(h^{2}\right)$ and $\tau_{5} \sim \mathcal{O}(1)$. So, the stabilized formulation in [17] mimics the regularized functional setting and cannot capture singular solutions.

As commented above, in this work we have considered stabilization terms of the standard variational multiscale (VMS) type. Straightforwardly, we could consider Galerkin least-square (GLS) terms (that only differ in some signs with respect to VMS), orthogonal sub-scale methods introducing the proper

$\mathcal{L}(x)=f$, where $\mathcal{L}$ is the differential operator, and with weak form $a(x, y)=(f, y)$, after the corresponding integrationby-parts and the definition of suitable functional spaces, the Galerkin formulation consists of replacing infinite-dimensional spaces by finite-dimensional ones, keeping the form of the problem, i.e. $a\left(x_{h}, y_{h}\right)=\left(f, y_{h}\right)$. The stabilized formulation we consider would replace this discrete problem by

$$
a\left(x_{h}, y_{h}\right)-\sum_{K \in \mathcal{T}_{h}} \int_{K} \mathcal{L}\left(x_{h}\right) \tau \mathcal{L}^{*}\left(y_{h}\right)=\left(f, y_{h}\right)-\sum_{K \in \mathcal{T}_{h}} \int_{K} f \tau \mathcal{L}^{*}\left(y_{h}\right),
$$

where $\tau(\mathbf{x})$ is the stabilization parameter, whose expression can be motivated using Fourier analysis [14] or inferred from the numerical analysis $[16,38]$.

${ }^{6} \tau_{5} \sim \mathcal{O}(1)$ would provide $\mathbf{b}_{h} \in H^{1}(\Omega)^{d}$ (see [3]) and so, it would not converge to singular solutions. 


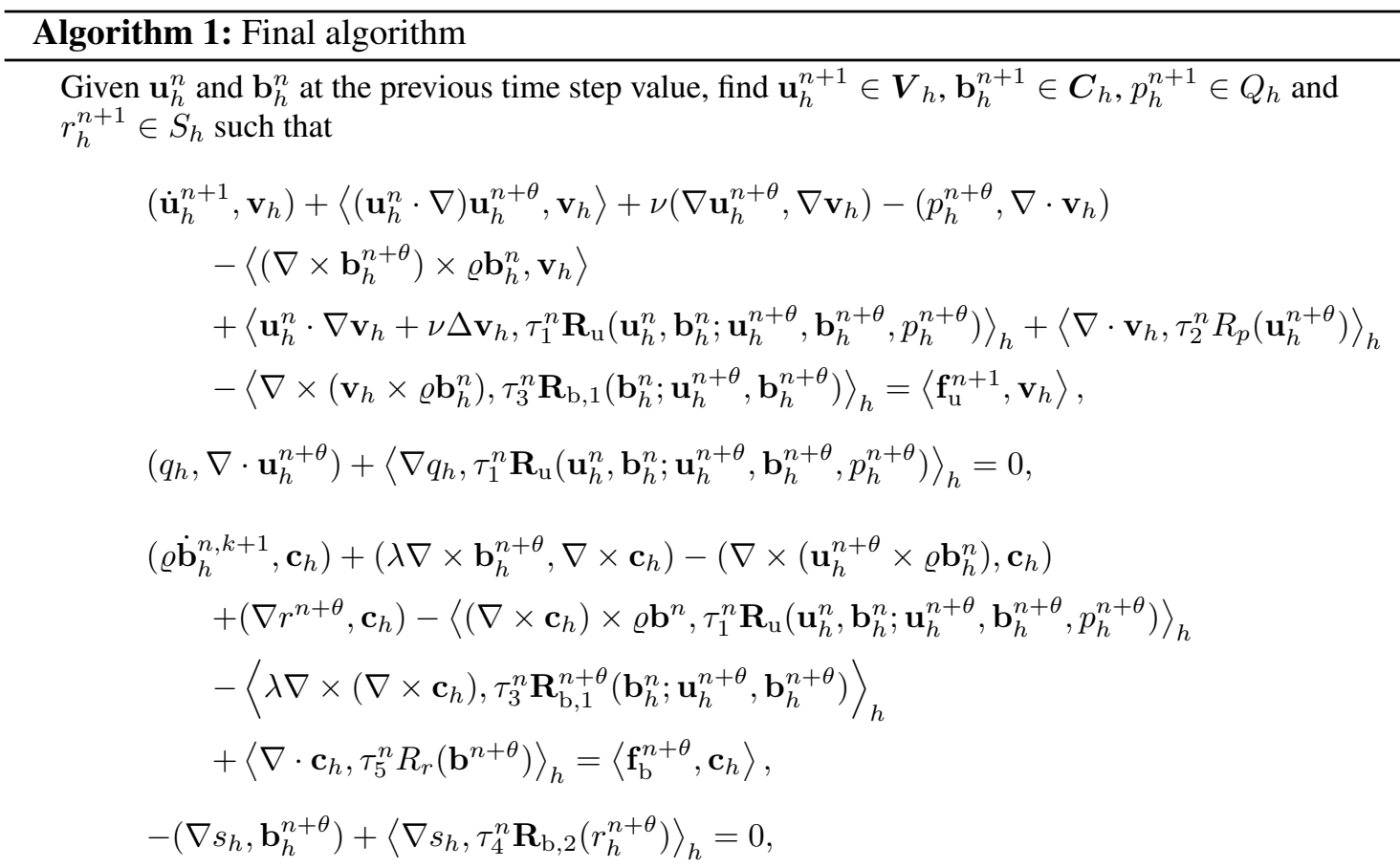

where the residuals are:

$$
\begin{array}{ll}
\mathbf{R}_{\mathrm{u}}\left(\mathbf{a}, \mathbf{d} ; \mathbf{u}_{h}, \mathbf{b}_{h}, p_{h}\right) & :=\dot{\mathbf{u}}_{h}+\mathbf{a} \cdot \nabla \mathbf{u}_{h}-\nu \Delta \mathbf{u}_{h}+\nabla p_{h}-\left(\nabla \times \mathbf{b}_{h}\right) \times \varrho \mathbf{d}-\mathbf{f}_{\mathrm{u}}, \\
R_{p}\left(\mathbf{u}_{h}\right) & :=\nabla \cdot \mathbf{u}_{h}, \\
\mathbf{R}_{\mathrm{b}, 1}\left(\mathbf{d} ; \mathbf{u}_{h}, \mathbf{b}_{h}\right) & :=\varrho \mathbf{b}_{h}+\lambda \nabla \times\left(\nabla \times \mathbf{b}_{h}\right)-\nabla \times\left(\mathbf{u}_{h} \times \varrho \mathbf{d}\right)-\mathbf{f}_{\mathrm{b}}, \\
\mathbf{R}_{\mathrm{b}, 2}\left(r_{h}\right) & :=\nabla r_{h}, \\
R_{r}\left(\mathbf{b}_{h}\right) & :=\nabla \cdot \mathbf{b}_{h} .
\end{array}
$$

The stabilization parameters have the following expressions within each element $K$ :

$$
\begin{array}{ll}
\tau_{1}^{m}:=\left(\alpha^{m}\right)^{-1}\left(1+\frac{\phi^{m}}{\sqrt{\alpha^{m} \gamma}}\right)^{-1}, & \tau_{2}:=c_{5} \frac{h^{2}}{\tau_{1}^{m}}, \quad \tau_{3}:=\gamma^{-1}\left(1+\frac{\phi^{m}}{\sqrt{\alpha^{m} \gamma}}\right)^{-1}, \\
\tau_{4}:=c_{6} \frac{L_{0}^{2}}{\lambda}, & \tau_{5}:=c_{7} \frac{h^{2} \lambda}{L_{0}^{2}},
\end{array}
$$

with

$$
\alpha^{m}:=c_{1} \frac{\left\|\mathbf{u}_{h}^{m}\right\|_{L^{\infty}(K)}}{h}+c_{2} \frac{\nu}{h^{2}}, \quad \phi^{m}:=c_{3} \frac{\varrho\left\|\mathbf{b}_{h}^{m}\right\|_{L^{\infty}(K)}}{h}, \quad \gamma:=c_{4} \frac{\lambda}{h^{2}} .
$$

$c_{1}, \ldots, c_{5}$ are algorithmic constants that must satisfy $c_{1}>\frac{2}{C_{\mathrm{inv}}^{2}}$ and $c_{3}>\frac{2}{C_{\mathrm{inv}}^{2}}$ and $L_{0}$ is a lengthscale of the problem.

projections [14] or more involved dynamic sub-grid closures [18]. In a subsequent work, we aim at exploiting these last approaches in the frame of MHD turbulence.

Remark 1. In the numerical analysis of the stabilized FE formulation for the Maxwell problem [3] and its extension to the full MHD system [5], convergence to singular solutions of of the magnetic field $\mathbf{b} \notin H^{1}(\Omega)^{d}$ is proved under one assumption about the approximability of the Lagrangian FE spaces. In particular, this assumption holds for meshes with a particular macro-element structure (see [3, Assumption 1 and Corollary 5]). One type of macro-element that satisfies this condition is the 
Powell-Sabin macro-element (see [43] and [10, Remark 4.1]). Further, in two dimensions, numerical experiments show that both the Powell-Sabin and "crossbox" elements provide excellent results. In the numerical experiments section, we extend this work to three-dimensions; we have considered both the 3D Powell-Sabin element and a 3D extension of the crossbox one; both choices exhibit excellent convergence properties. Finally, let us stress the fact that this macro-element structure is only needed for singular solutions, that appear in non-convex domains. For smooth solutions, with $\mathbf{b} \in H^{1}(\Omega)^{d}$, the convergence analysis and approximability properties are easy to check and hold for any type of mesh. We refer to [3] for a detailed discussion on this topic, in the framework of the Maxwell operator. Further, in [2] we have observed that the method still converges to singular solutions for general meshes with a significantly lower order of convergence.

\section{Numerical experimentation}

The numerical examples shown in the next subsections have been computed with some common computational settings. First, linear $\mathcal{C}^{0}$ Lagrangian FEs have been used, both in the 2D and 3D cases. $^{7}$ The solution of the resulting linear systems of equations has been obtained via a direct solver. The problem non-linearity has been solved through Picard iterations until converging to a tolerance of $10^{-4}$. Finally, the constants that appear in the definition of the stabilization parameters in Algorithm 1 have been chosen as:

$$
c_{1}=c_{3}=2, \quad c_{2}=c_{4}=c_{7}=4, \quad \text { and } \quad c_{5}=c_{6}=1 .
$$

\subsection{Convergence to singular solutions. Case 2D}

The stabilization method presented in the previous chapter has been used to solve the MHD problem in a nonconvex L-shaped domain. Due to the re-entrant corner present in the domain, both the Stokes and the Maxwell operators have strong singularities at this corner, where the origin of coordinates is taken. The non-smooth solution for the Stokes operator is described in polar coordinates $(r, \theta)$ by

$$
\begin{aligned}
u_{x}(x, y) & =r^{\lambda}\left((1+\lambda) \sin (\theta) \psi(\theta)+\cos (\theta) \psi^{\prime}(\theta)\right), \\
u_{y}(x, y) & =r^{\lambda}\left(-(1+\lambda) \cos (\theta) \psi(\theta)+\sin (\theta) \psi^{\prime}(\theta)\right), \\
p(x, y) & =-\frac{r^{\lambda-1}}{1-\lambda}\left((1+\lambda)^{2} \psi^{\prime}(\theta)+\psi^{\prime \prime \prime}(\theta)\right),
\end{aligned}
$$

where

$$
\psi(\theta)=\sin ((1+\lambda) \theta) \frac{\cos (\lambda \omega)}{1+\lambda}-\cos ((1+\lambda) \theta)-\sin ((1-\lambda) \theta) \frac{\cos (\lambda \omega)}{1-\lambda}+\cos ((1-\lambda) \theta) .
$$

The value of the parameter $\lambda$ is the smallest positive solution of

$$
\sin (\lambda \omega)+\lambda \sin (\omega)=0, \quad \text { where } \omega=\frac{3 \pi}{2},
$$

\footnotetext{
${ }^{7}$ In this situation, all the system matrix blocks have the sparsity pattern defined by the mesh graph.
} 


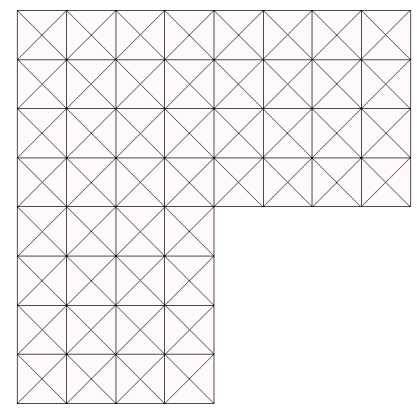

(a) Crossbox element

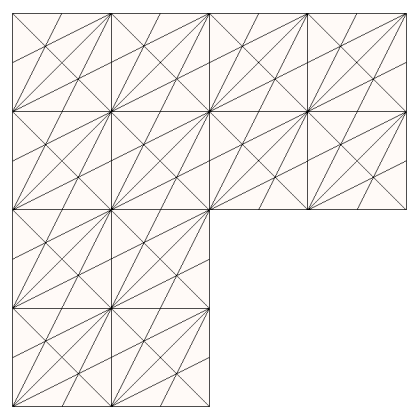

(b) Powell-Sabin element

Figure 1: Mesh structures for the 2D case.

which is $\lambda \sim 0.54448373678246$. Note that $\mathbf{u}=\left(u_{x}, u_{y}\right)$ is solenoidal and $(\mathbf{u}, p) \in H^{1+\lambda}(\Omega)^{2} \times$ $H^{\lambda}(\Omega)$.

The singular solution for the Maxwell operator is defined also in polar coordinates as

$$
\mathbf{b}(x, y)=\nabla\left(r^{\frac{2 n}{3}} \sin \left(\frac{2 n}{3} \theta\right)\right), \quad n \in \mathbb{N}^{+} .
$$

Note that $\nabla \cdot \mathbf{b}=0$ and $\nabla \times \mathbf{b}=\mathbf{0}$. The magnetic induction field $\mathbf{b} \in H^{\frac{2 n}{3}}(\Omega)^{2}$ and therefore, $\mathbf{b} \notin H^{1}(\Omega)^{2}$ for $n=1$ only.

The computation has been done fully-coupled, solving the implicit scheme stated in Algorithm 1 and using two different mesh structures suitable to approximate the Maxwell operator singularity, namely the Powell-Sabin and the crossbox element. Figure 1 shows an example of the two different mesh structures.

Remark 2. Let us stress the fact that, since both crossbox and Powell-Sabin macro-element meshes allow to converge to singular solutions, we can start with any triangle or quadrilateral mesh, and after a simple processing of the mesh, solve the problem on the modified mesh. Therefore, the previous macro-element topology can be attained for any original mesh.

In both cases, the problem has been solved on several meshes with different mesh sizes, from the coarsest one with $h=2^{-2}$ to the finest one with $h=2^{-7}$. Tables 2 and 3 contain the numerical error norms for the case of crossbox element meshes. In Table 2, we show the numerical errors for the velocity in the $L^{2}$-norm and the $H^{1}$-norm and the error for the pressure in the $L^{2}$-norm. In Table 3 , we have listed the numerical errors for the magnetic unknowns, that is, the error for the magnetic induction in the $L^{2}$-norm and the $H(\mathbf{c u r l})$-norm and the errors for the magnetic pseudo-pressure in both the $L^{2}$-norm and the $H^{1}$-norm.

Furthermore, Figure 2 shows the convergence plots of the numerical errors presented in Tables 2 and 3. It is clearly seen that the proposed method is able to converge to the singular solutions when using meshes of crossbox elements. 


\begin{tabular}{|c|c|c|c|}
\hline$h$ & $\left\|\mathbf{e}_{\mathrm{u}}\right\|$ & $\left\|\nabla \mathbf{e}_{\mathrm{u}}\right\|$ & $\left\|e_{p}\right\|$ \\
\hline \hline $2^{-2}$ & $8.49 \cdot 10^{-2}(-)$ & $1.30 \cdot 10^{0}(-)$ & $1.69 \cdot 10^{0}(-)$ \\
$2^{-3}$ & $3.45 \cdot 10^{-2}(1.30)$ & $8.95 \cdot 10^{-1}(0.54)$ & $1.13 \cdot 10^{0}(0.58)$ \\
$2^{-4}$ & $1.45 \cdot 10^{-2}(1.25)$ & $6.16 \cdot 10^{-1}(0.54)$ & $7.66 \cdot 10^{-1}(0.56)$ \\
$2^{-5}$ & $6.28 \cdot 10^{-3}(1.21)$ & $4.24 \cdot 10^{-1}(0.54)$ & $5.22 \cdot 10^{-1}(0.55)$ \\
$2^{-6}$ & $2.79 \cdot 10^{-3}(1.17)$ & $2.91 \cdot 10^{-1}(0.54)$ & $3.57 \cdot 10^{-1}(0.55)$ \\
$2^{-7}$ & $1.27 \cdot 10^{-3}(1.14)$ & $2.00 \cdot 10^{-1}(0.54)$ & $2.44 \cdot 10^{-1}(0.55)$ \\
\hline
\end{tabular}

Table 2: Numerical errors for hydrodynamic unknowns and rate of convergence in brackets. Crossbox element in 2D.

\begin{tabular}{|c|c|c|c|c|}
\hline$h$ & $\left\|\mathbf{e}_{\mathrm{b}}\right\|$ & $\left\|\nabla \times \mathbf{e}_{\mathrm{b}}\right\|$ & $\left\|e_{r}\right\|$ & $\left\|\nabla e_{r}\right\|$ \\
\hline \hline $2^{-2}$ & $4.01 \cdot 10^{-1}(-)$ & $4.19 \cdot 10^{-1}(-)$ & $9.34 \cdot 10^{-2}(-)$ & $3.77 \cdot 10^{-1}(-)$ \\
$2^{-3}$ & $2.67 \cdot 10^{-1}(0.59)$ & $2.12 \cdot 10^{-1}(0.98)$ & $5.21 \cdot 10^{-2}(0.84)$ & $2.83 \cdot 10^{-1}(0.41)$ \\
$2^{-4}$ & $1.73 \cdot 10^{-1}(0.63)$ & $1.04 \cdot 10^{-1}(1.03)$ & $2.45 \cdot 10^{-2}(1.09)$ & $1.93 \cdot 10^{-1}(0.55)$ \\
$2^{-5}$ & $1.10 \cdot 10^{-1}(0.65)$ & $4.94 \cdot 10^{-2}(1.07)$ & $1.06 \cdot 10^{-2}(1.21)$ & $1.26 \cdot 10^{-1}(0.62)$ \\
$2^{-6}$ & $6.94 \cdot 10^{-2}(0.66)$ & $2.29 \cdot 10^{-2}(1.11)$ & $4.42 \cdot 10^{-3}(1.26)$ & $8.02 \cdot 10^{-2}(0.65)$ \\
$2^{-7}$ & $4.38 \cdot 10^{-2}(0.66)$ & $1.05 \cdot 10^{-2}(1.12)$ & $1.80 \cdot 10^{-3}(1.30)$ & $5.07 \cdot 10^{-2}(0.66)$ \\
\hline
\end{tabular}

Table 3: Numerical errors for magnetic unknowns and rate of convergence in brackets. Crossbox element in 2D.

Tables 4 and 5 display the numerical errors obtained when using meshes with a Powell-Sabin macroelement structure to approximate the singular solutions. We show in these tables the same error quantities as before, for the fluid and the magnetic sub-problems respectively.

Figure 3 shows the convergence plots of the numerical errors presented in Tables 4 and 5. Similarly to the previous case, the proposed method with a Powell-Sabin mesh is clearly suitable to approximate non-smooth solutions for both the Stokes and the Maxwell operator.

\subsection{Convergence to singular solutions. Extension to the $3 D$ case}

The easiest problem with singular solutions in dimension three consists of the two-dimensional solution for the $x-y$ components and a constant in the third component. In this case, the third component ( $z$-axis) and its corresponding derivatives are null. Therefore, the computational domain considered is the volume generated by the extrusion of the 2D L-shaped domain ( $x-y$ plane) in the $z$-axis, for a given thickness. Periodic boundary conditions have been set in the planes $x-y$.

Analogously to the bi-dimensional case, the approximation of non-smooth solutions of the Maxwell operator requires solving the problem on a proper modification of the original mesh with a macroelement structure. In the three-dimensional case, the 3D extensions of the bi-dimensional crossbox and the Powell-Sabin macro-elements displayed in Figure 4 are suitable to converge to the singular solutions. On one hand, the Powell-Sabin macro-element in 3D consists of the division of every 


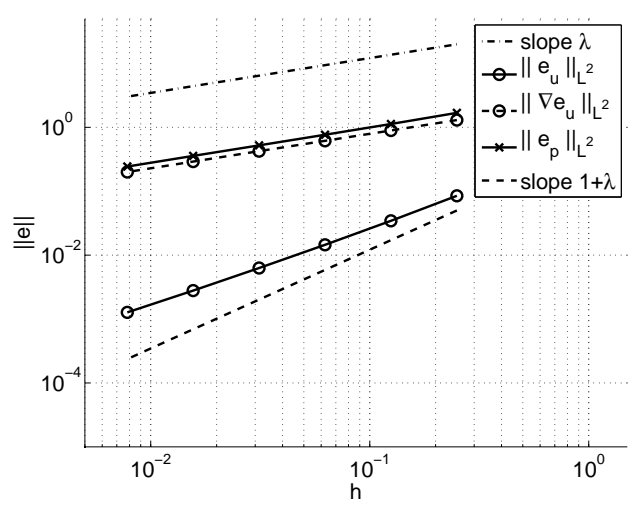

(a) Hydrodynamic unknowns

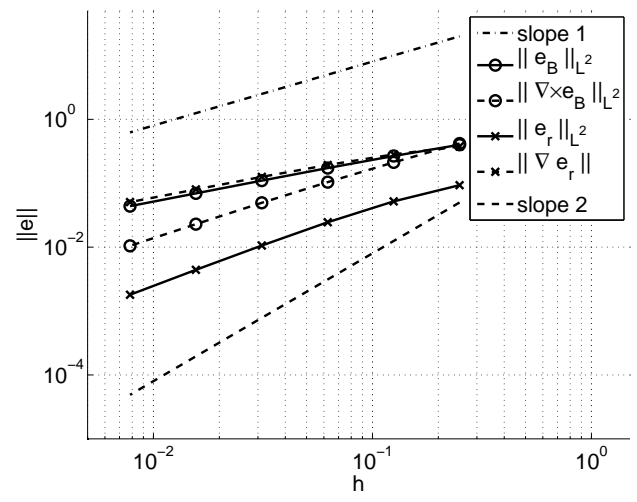

(b) Magnetic unknowns

Figure 2: Convergence plots for the singular solutions in the 2D case. Crossbox element.

\begin{tabular}{|c|c|c|c|}
\hline$h$ & $\left\|\mathbf{e}_{\mathrm{u}}\right\|$ & $\left\|\nabla \mathbf{e}_{\mathrm{u}}\right\|$ & $\left\|e_{p}\right\|$ \\
\hline \hline $2^{-2}$ & $1.15 \cdot 10^{-1}(-)$ & $1.35 \cdot 10^{0}(-)$ & $2.00 \cdot 10^{0}(-)$ \\
$2^{-3}$ & $4.61 \cdot 10^{-2}(1.32)$ & $9.35 \cdot 10^{-1}(0.53)$ & $1.31 \cdot 10^{0}(0.61)$ \\
$2^{-4}$ & $1.89 \cdot 10^{-2}(1.29)$ & $6.45 \cdot 10^{-1}(0.54)$ & $8.79 \cdot 10^{-1}(0.58)$ \\
$2^{-5}$ & $7.99 \cdot 10^{-3}(1.24)$ & $4.44 \cdot 10^{-1}(0.54)$ & $5.97 \cdot 10^{-1}(0.56)$ \\
$2^{-6}$ & $3.49 \cdot 10^{-3}(1.19)$ & $3.05 \cdot 10^{-1}(0.54)$ & $4.07 \cdot 10^{-1}(0.55)$ \\
$2^{-7}$ & $1.56 \cdot 10^{-3}(1.16)$ & $2.10 \cdot 10^{-1}(0.54)$ & $2.79 \cdot 10^{-1}(0.54)$ \\
\hline
\end{tabular}

Table 4: Numerical errors for hydrodynamic unknowns and rate of convergence in brackets. Powell-Sabin element in 2D.

\begin{tabular}{|c|c|c|c|c|}
\hline$h$ & $\left\|\mathbf{e}_{\mathrm{b}}\right\|$ & $\left\|\nabla \times \mathbf{e}_{\mathrm{b}}\right\|$ & $\left\|e_{r}\right\|$ & $\left\|\nabla e_{r}\right\|$ \\
\hline \hline $2^{-2}$ & $4.51 \cdot 10^{-1}(-)$ & $5.11 \cdot 10^{-1}(-)$ & $1.04 \cdot 10^{-1}(-)$ & $3.95 \cdot 10^{-1}(-)$ \\
$2^{-3}$ & $3.04 \cdot 10^{-1}(0.57)$ & $2.61 \cdot 10^{-1}(0.97)$ & $6.24 \cdot 10^{-2}(0.74)$ & $3.09 \cdot 10^{-1}(0.35)$ \\
$2^{-4}$ & $1.97 \cdot 10^{-1}(0.63)$ & $1.29 \cdot 10^{-1}(1.02)$ & $3.02 \cdot 10^{-2}(1.05)$ & $2.15 \cdot 10^{-1}(0.52)$ \\
$2^{-5}$ & $1.26 \cdot 10^{-1}(0.64)$ & $6.19 \cdot 10^{-2}(1.06)$ & $1.33 \cdot 10^{-2}(1.18)$ & $1.41 \cdot 10^{-1}(0.61)$ \\
$2^{-6}$ & $7.98 \cdot 10^{-2}(0.66)$ & $2.89 \cdot 10^{-2}(1.10)$ & $5.54 \cdot 10^{-3}(1.26)$ & $9.04 \cdot 10^{-2}(0.64)$ \\
$2^{-7}$ & $5.03 \cdot 10^{-2}(0.67)$ & $1.33 \cdot 10^{-2}(1.12)$ & $2.26 \cdot 10^{-3}(1.29)$ & $5.70 \cdot 10^{-2}(0.67)$ \\
\hline
\end{tabular}

Table 5: Numerical errors for magnetic unknowns and rate of convergence in brackets. Powell-Sabin element in 2D. 


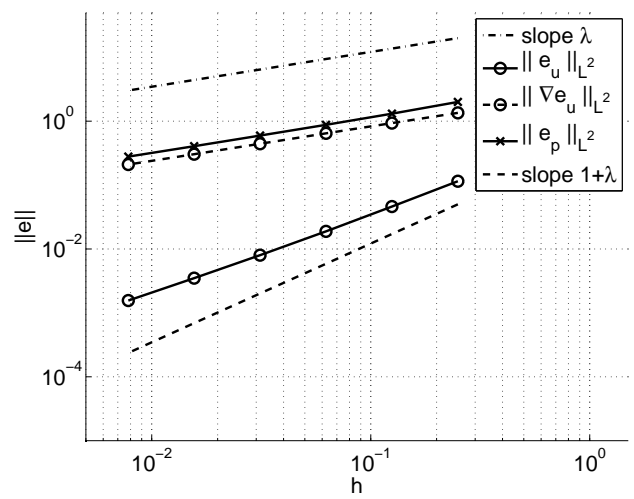

(a) Hydrodynamic unknowns

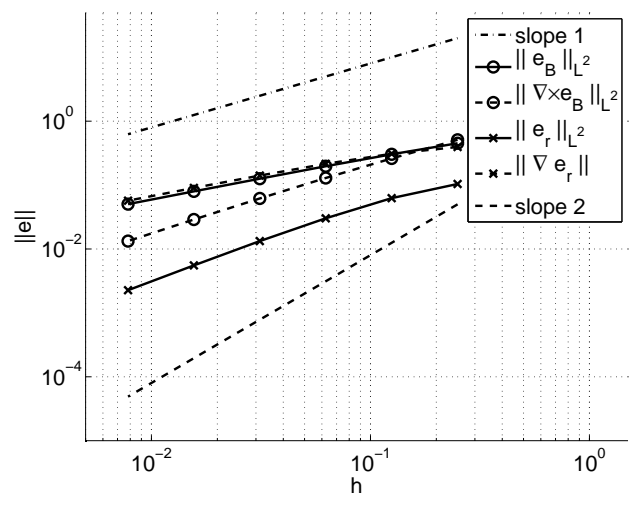

(b) Magnetic unknowns

Figure 3: Convergence plots for the singular solutions in the 2D case. Powell-Sabin element.

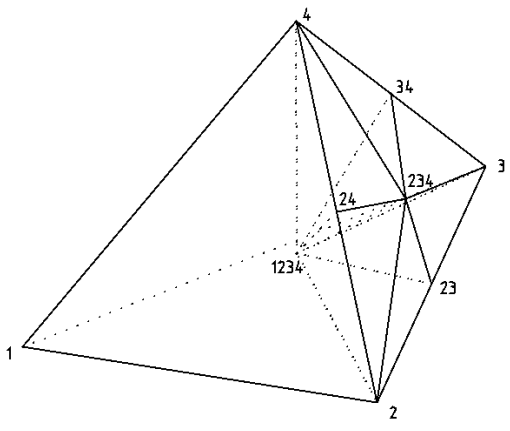

(a) Tetrahedron remeshing

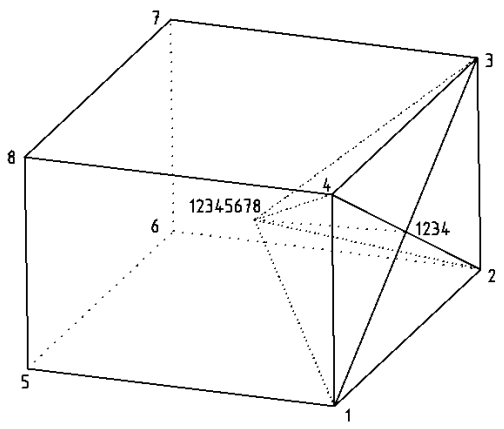

(b) Hexahedron remeshing

Figure 4: Remeshing structure suitable for interpolation of Maxwell singular solutions.

tetrahedron of the original mesh into 24 new tetrahedra [43]. Each tetrahedron face is decomposed into 6 parts as the Powell-Sabin element in 2D. Each one of these parts forms a tetrahedron together with the original tetrahedron centroid (see Figure 4(a)). In this way, 6 new tetrahedra are obtained for each one of the original tetrahedron faces. On the other hand, the extension to three dimensions of the crossbox element requires an original (possibly) non-structured mesh of hexahedra. Each hexahedron face is divided into 4 triangles as the crossbox element in 2D and then, 4 tetrahedra are obtained together with the hexahedron centroid (see Figure 4(b)). Therefore, each original hexahedron is divided into 24 new tetrahedra.

Remark 3. An equivalent remark to the bi-dimensional case can be made. In three dimensions, we can use any tetrahedral or hexahedral mesh and easily process this mesh in order to get one with the $3 D$ crossbox or $3 D$ Powell-Sabin macro-element structure.

The results obtained for different mesh-sizes using crossbox elements in 3D are displayed in Tables 


\begin{tabular}{|c|c|c|c|}
\hline$h$ & $\left\|\mathbf{e}_{\mathrm{u}}\right\|$ & $\left\|\nabla \mathbf{e}_{\mathrm{u}}\right\|$ & $\left\|e_{p}\right\|$ \\
\hline \hline $2^{-2}$ & $2.30 \cdot 10^{-2}(-)$ & $3.08 \cdot 10^{-1}(-)$ & $4.49 \cdot 10^{-1}(-)$ \\
$2^{-3}$ & $9.04 \cdot 10^{-3}(1.35)$ & $2.06 \cdot 10^{-1}(0.58)$ & $3.19 \cdot 10^{-1}(0.49)$ \\
$2^{-4}$ & $3.34 \cdot 10^{-3}(1.44)$ & $1.37 \cdot 10^{-1}(0.59)$ & $2.21 \cdot 10^{-1}(0.53)$ \\
$2^{-5}$ & $1.19 \cdot 10^{-3}(1.49)$ & $8.99 \cdot 10^{-2}(0.61)$ & $1.52 \cdot 10^{-1}(0.54)$ \\
$2^{-6}$ & $4.19 \cdot 10^{-4}(1.51)$ & $5.94 \cdot 10^{-2}(0.60)$ & $1.04 \cdot 10^{-1}(0.55)$ \\
\hline
\end{tabular}

Table 6: Numerical errors for hydrodynamic unknowns and rate of convergence in brackets. Crossbox element in 3D.

\begin{tabular}{|c|c|c|c|c|}
\hline$h$ & $\left\|\mathbf{e}_{\mathrm{b}}\right\|$ & $\left\|\nabla \times \mathbf{e}_{\mathrm{b}}\right\|$ & $\left\|e_{r}\right\|$ & $\left\|\nabla e_{r}\right\|$ \\
\hline \hline $2^{-2}$ & $1.04 \cdot 10^{-1}(-)$ & $1.05 \cdot 10^{-1}(-)$ & $2.31 \cdot 10^{-1}(-)$ & $8.31 \cdot 10^{-1}(-)$ \\
$2^{-3}$ & $7.05 \cdot 10^{-2}(0.56)$ & $5.06 \cdot 10^{-2}(1.05)$ & $1.68 \cdot 10^{-1}(0.46)$ & $7.38 \cdot 10^{-1}(0.17)$ \\
$2^{-4}$ & $4.61 \cdot 10^{-2}(0.61)$ & $2.21 \cdot 10^{-2}(1.20)$ & $8.87 \cdot 10^{-2}(0.92)$ & $5.37 \cdot 10^{-1}(0.46)$ \\
$2^{-5}$ & $2.95 \cdot 10^{-2}(0.64)$ & $9.24 \cdot 10^{-3}(1.26)$ & $4.07 \cdot 10^{-2}(1.12)$ & $3.61 \cdot 10^{-1}(0.57)$ \\
$2^{-6}$ & $1.87 \cdot 10^{-2}(0.66)$ & $3.79 \cdot 10^{-3}(1.29)$ & $1.75 \cdot 10^{-2}(1.22)$ & $2.35 \cdot 10^{-1}(0.62)$ \\
\hline
\end{tabular}

Table 7: Numerical errors for magnetic unknowns and rate of convergence in brackets. Crossbox element in 3D.

6 and 7. The error norms calculated are the same as in the 2D cases. It is clearly seen that, similarly to the $2 \mathrm{D}$ case, the numerical solution is able to converge to singular (non-smooth) solutions. Figure 5 shows graphically the convergence plots displaying the obtained slopes for every computed error norm.

The results obtained when using the Powell-Sabin elements in 3D instead of the crossbox elements are shown in Tables 8 and 9. The error norms computed in this case are the same as in the previous case with crossbox elements, and the behavior of the numerical solution is the same. The results show clearly that the proposed method is able to converge to singular solutions. Figure 6 displays the convergence plots showing graphically the obtained results.

\begin{tabular}{|c|c|c|c|}
\hline$h$ & $\left\|\mathbf{e}_{\mathrm{u}}\right\|$ & $\left\|\nabla \mathbf{e}_{\mathrm{u}}\right\|$ & $\left\|e_{p}\right\|$ \\
\hline \hline $2^{-2}$ & $4.18 \cdot 10^{-2}(-)$ & $4.41 \cdot 10^{-1}(-)$ & $5.71 \cdot 10^{-1}(-)$ \\
$2^{-3}$ & $1.40 \cdot 10^{-2}(1.58)$ & $2.64 \cdot 10^{-1}(0.74)$ & $3.41 \cdot 10^{-1}(0.74)$ \\
$2^{-4}$ & $4.51 \cdot 10^{-3}(1.63)$ & $1.59 \cdot 10^{-1}(0.73)$ & $2.24 \cdot 10^{-1}(0.61)$ \\
$2^{-5}$ & $1.53 \cdot 10^{-3}(1.56)$ & $1.00 \cdot 10^{-1}(0.67)$ & $1.52 \cdot 10^{-1}(0.56)$ \\
$2^{-6}$ & $5.53 \cdot 10^{-4}(1.47)$ & $6.63 \cdot 10^{-2}(0.59)$ & $1.03 \cdot 10^{-1}(0.56)$ \\
\hline
\end{tabular}

Table 8: Numerical errors for hydrodynamic unknowns and rate of convergence in brackets. Powell-Sabin element in 3D. 


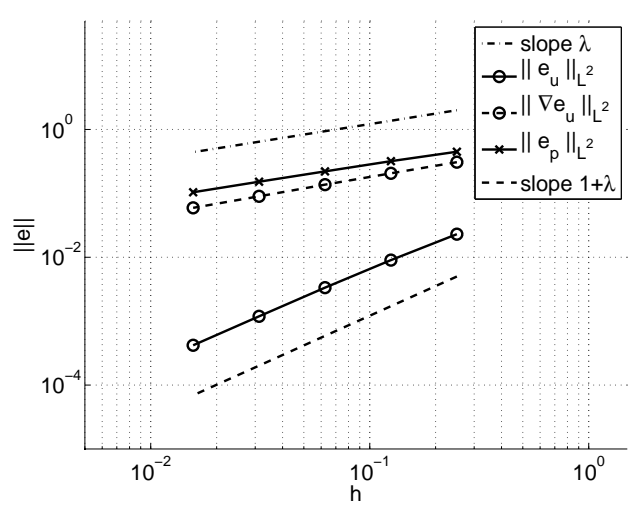

(a) Hydrodynamic unknowns

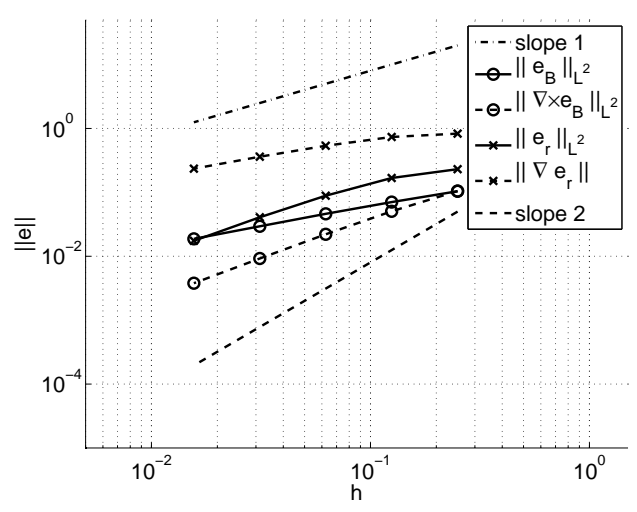

(b) Magnetic unknowns

Figure 5: Convergence plots for the singular solutions in the 3D case. Crossbox element.

\begin{tabular}{|c|c|c|c|c|}
\hline$h$ & $\left\|\mathbf{e}_{\mathrm{b}}\right\|$ & $\left\|\nabla \times \mathbf{e}_{\mathrm{b}}\right\|$ & $\left\|e_{r}\right\|$ & $\left\|\nabla e_{r}\right\|$ \\
\hline \hline $2^{-2}$ & $1.22 \cdot 10^{-1}(-)$ & $1.20 \cdot 10^{-1}(-)$ & $2.58 \cdot 10^{-1}(-)$ & $9.08 \cdot 10^{-1}(-)$ \\
$2^{-3}$ & $8.60 \cdot 10^{-2}(0.50)$ & $6.17 \cdot 10^{-2}(0.96)$ & $2.02 \cdot 10^{-1}(0.35)$ & $8.65 \cdot 10^{-1}(0.07)$ \\
$2^{-4}$ & $5.76 \cdot 10^{-2}(0.58)$ & $2.90 \cdot 10^{-2}(1.09)$ & $1.12 \cdot 10^{-1}(0.85)$ & $6.64 \cdot 10^{-1}(0.38)$ \\
$2^{-5}$ & $3.75 \cdot 10^{-2}(0.62)$ & $1.28 \cdot 10^{-2}(1.18)$ & $5.28 \cdot 10^{-2}(1.08)$ & $4.60 \cdot 10^{-1}(0.53)$ \\
$2^{-6}$ & $2.40 \cdot 10^{-2}(0.64)$ & $5.26 \cdot 10^{-3}(1.28)$ & $2.29 \cdot 10^{-2}(1.21)$ & $3.02 \cdot 10^{-1}(0.61)$ \\
\hline
\end{tabular}

Table 9: Numerical errors for magnetic unknowns and rate of convergence in brackets. Powell-Sabin element in 3D.

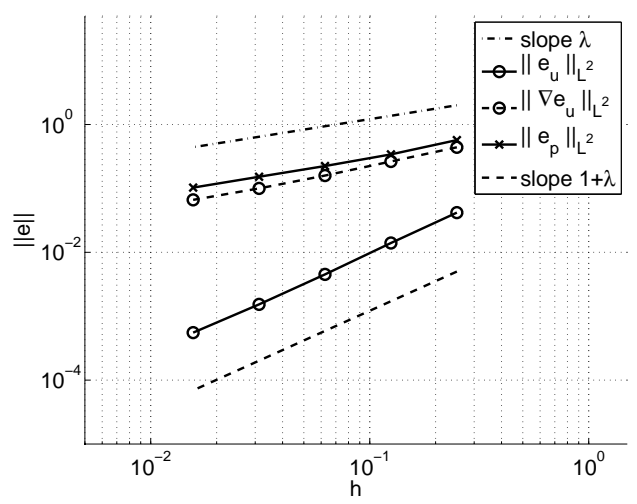

(a) Hydrodynamic unknowns

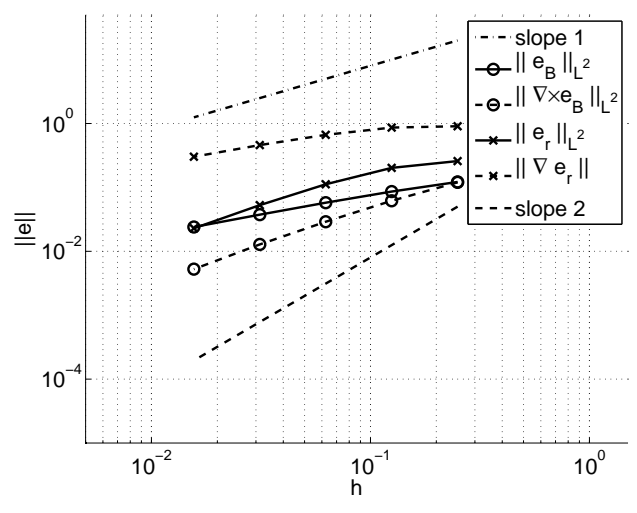

(b) Magnetic unknowns

Figure 6: Convergence plots for the singular solutions in the 3D case. Powell-Sabin element. 


\subsection{Classical MHD problems with analytical solution. Shercliff's case}

The stabilized finite element method proposed in this paper has also been tested to simulate some classical problems of MHD, namely Shercliff's [42] and Hunt's [32] cases. Shercliff's problem consists of a fully developed flow in a squared-section channel where both the Hartmann walls, perpendicular to the externally applied magnetic field, and the side walls, parallel to the external magnetic field, are considered electrically insulating. Considering that the external magnetic field, $\mathbf{b}_{\text {ext }}$ is applied in the $y$-direction and the longitudinal axis of the channel is the $z$-direction, the fluid flows driven by an imposed pressure gradient, with a velocity field where only the $z$-component is not null. This problem has an analytical solution in the form of Fourier series, whose expression can be found in $[42,38]$.

The computational domain has been set as a channel slice of width $1 / 100$ times the section sides. The boundary conditions on the inflow and outflow sections have been set as periodic conditions in order to enforce the condition of fully developed flow. The pressure gradient that drives the flow has been set as an external hydrodynamic body force, with value

$$
\begin{gathered}
\frac{\mathrm{d} p}{\mathrm{~d} z}=\frac{K L^{3}}{\rho \nu^{2} \mathrm{Re}} \\
K=\frac{\mathrm{Ha}}{1-0.825 \mathrm{Ha}^{-1 / 2}-\mathrm{Ha}^{-1}}, \quad \mathrm{Re}=\frac{\mathrm{UL}}{\nu},
\end{gathered}
$$

where Ha and Re are the Hartmann ${ }^{8}$ and Reynolds ${ }^{9}$ numbers, respectively.

Finally, the boundary conditions on the walls, both the Hartmann and side walls, have been set as non-slip conditions for the velocity, $\mathbf{u}=\mathbf{0}$, and as the essential boundary conditions for the magnetic unknowns because the walls are electrically insulating,

$$
\mathbf{b} \times \mathbf{n}=\mathbf{b}_{\text {ext }} \times \mathbf{n} \quad \text { and } \quad r=0 .
$$

The physical properties of the problem, density, viscosity, electrical conductivity and magnetic permeability have been set to one when solving this problem. Therefore, the Hartmann number Ha corresponds to the norm of the externally applied magnetic field. This problem has been solved on several meshes with different mesh sizes $h$ in order to study the convergence properties of the method. Two different cases have been simulated depending on the Hartmann number, namely $\mathrm{Ha}=10$ and $\mathrm{Ha}=100$, in order to show that the method has optimal convergence properties independently of the coupling between the hydrodynamic and the magnetic problems.

${ }^{8}$ The Hartmann number is the ratio between electromagnetic and viscous forces. Its expression is:

$$
\mathrm{Ha}=B L \sqrt{\frac{\sigma}{\rho \nu}},
$$

where $L$ is a characteristic length of the problem.

${ }^{9}$ The Reynolds number is the ratio between inertial and viscous forces in a fluid. It can be computed from:

$$
\operatorname{Re}=\frac{U L}{\nu},
$$

where $L$ is a characteristic length and $U$ is a characteristic velocity of the problem. 


\begin{tabular}{|c|c|c|c|c|}
\hline$h$ & $\left\|\mathbf{e}_{\mathrm{u}}\right\|$ & $\left\|\nabla \mathbf{e}_{\mathrm{u}}\right\|$ & $\left\|\mathbf{e}_{\mathrm{b}}\right\|$ & $\left\|\nabla \times \mathbf{e}_{\mathrm{b}}\right\|$ \\
\hline \hline $2^{-2}$ & $3.32 \cdot 10^{-3}(-)$ & $5.62 \cdot 10^{-2}(-)$ & $1.90 \cdot 10^{-3}(-)$ & $4.20 \cdot 10^{-2}(-)$ \\
$2^{-3}$ & $1.25 \cdot 10^{-3}(1.41)$ & $3.19 \cdot 10^{-2}(0.82)$ & $5.01 \cdot 10^{-4}(1.92)$ & $2.13 \cdot 10^{-2}(0.98)$ \\
$2^{-4}$ & $3.73 \cdot 10^{-4}(1.74)$ & $1.56 \cdot 10^{-2}(1.03)$ & $1.59 \cdot 10^{-4}(1.66)$ & $1.08 \cdot 10^{-2}(0.98)$ \\
$2^{-5}$ & $9.72 \cdot 10^{-5}(1.94)$ & $6.88 \cdot 10^{-3}(1.18)$ & $6.13 \cdot 10^{-5}(1.38)$ & $5.43 \cdot 10^{-3}(0.99)$ \\
\hline
\end{tabular}

Table 10: Numerical errors and rate of convergence in brackets. Shercliff's case with $\mathrm{Ha}=10$.

\begin{tabular}{|c|c|c|c|c|}
\hline$h^{*}$ & $\left\|\mathbf{e}_{\mathrm{u}}\right\|$ & $\left\|\nabla \mathbf{e}_{\mathrm{u}}\right\|$ & $\left\|\mathbf{e}_{\mathrm{b}}\right\|$ & $\left\|\nabla \times \mathbf{e}_{\mathrm{b}}\right\|$ \\
\hline \hline $2^{-2}$ & $6.65 \cdot 10^{-3}(-)$ & $1.43 \cdot 10^{-1}(-)$ & $5.70 \cdot 10^{-3}(-)$ & $1.29 \cdot 10^{-1}(-)$ \\
$2^{-3}$ & $2.40 \cdot 10^{-3}(1.47)$ & $1.37 \cdot 10^{-1}(0.06)$ & $2.06 \cdot 10^{-3}(1.47)$ & $1.26 \cdot 10^{-1}(0.03)$ \\
$2^{-4}$ & $3.99 \cdot 10^{-4}(2.59)$ & $3.74 \cdot 10^{-2}(1.87)$ & $3.88 \cdot 10^{-4}(2.41)$ & $3.59 \cdot 10^{-2}(1.81)$ \\
$2^{-5}$ & $1.66 \cdot 10^{-5}(4.59)$ & $2.00 \cdot 10^{-3}(4.22)$ & $7.13 \cdot 10^{-5}(2.44)$ & $2.07 \cdot 10^{-3}(4.12)$ \\
\hline
\end{tabular}

Table 11: Numerical errors and rate of convergence in brackets. Shercliff's case with Ha=100.

On one hand, Shercliff's case has been solved for $\mathrm{Ha}=10$ and Reynolds number $\mathrm{Re}=10$ on a series of uniformly structured meshes composed of linear tetrahedral elements. The obtained results are shown in Table 10. The error norms computed correspond to the $L^{2}$-norm of the velocity error $\left\|\mathbf{e}_{\mathrm{u}}\right\|$, the velocity gradient error $\left\|\nabla \mathbf{e}_{\mathrm{u}}\right\|$, the magnetic induction error $\left\|\mathbf{e}_{\mathrm{b}}\right\|$ and the curl of the magnetic induction error $\left\|\nabla \times \mathbf{e}_{\mathrm{b}}\right\|$. The number in brackets is the rate of convergence. The same results have been plotted in Figure 7(a), where it can be clearly seen that the expected optimal order of convergence is achieved.

The same problem has been solved for $\mathrm{Ha}=100$ and $\mathrm{Re}=10$. In this case, the meshes used for the computations are also structured meshes of linear tetrahedra but concentrating the elements near the walls to be able to capture the sharper boundary layers that appear in this case. Therefore, the mesh size $h^{*}$ used in the convergence study of this problem corresponds to the mesh size of a uniformly structured mesh with the same number of degrees of freedom. Similarly to the previous case, the computed error norms are displayed in Table 11. Figure 7(b) shows graphically the behavior of the error norms with $h^{*}$. It is also very clear that the proposed method has very good convergence properties in this case.

\subsection{Classical MHD problems with analytical solution. Hunt's case}

Hunt's problem simulates a fully developed flow in a channel with square section under an external magnetic field $\mathbf{b}_{\text {ext }}$. In this case, the Hartmann walls are considered to be perfectly conducting whereas the side walls are electrically insulating. Similarly to the previous Shercliff's case, this problem has an analytical solution in terms of Fourier series, whose expression can be found in $[32,38]$.

The computational domain where this problem has been solved is the same that in Shercliff's case, see 


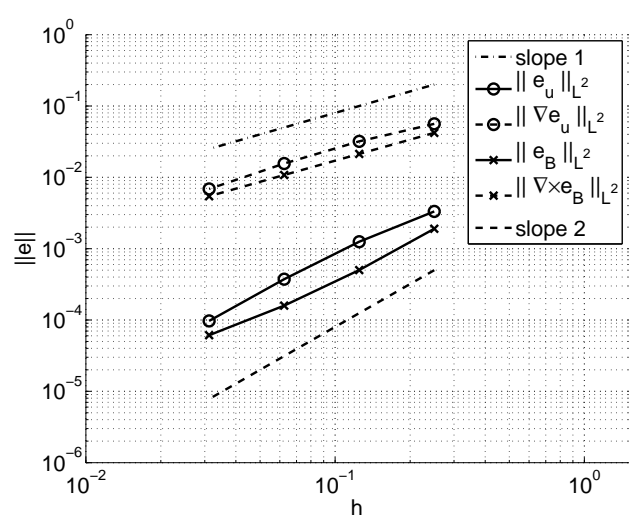

(a) $\mathrm{Ha}=10$

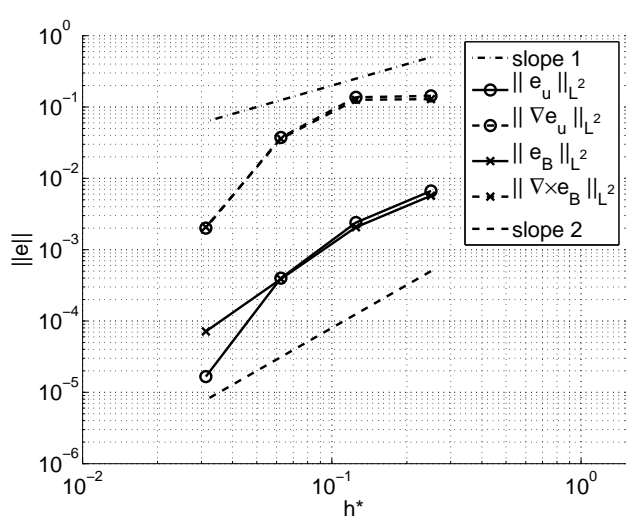

(b) $\mathrm{Ha}=100$

Figure 7: Convergence plots of velocity and induction error norms for Shercliff's case.

Section 6.3. Similarly, the boundary conditions on the inflow and outflow have been set to periodic conditions. In both the Hartmann walls and side walls, the hydrodynamic boundary conditions have been set as the non-slip condition, $\mathbf{u}=\mathbf{0}$. The magnetic boundary conditions for the side walls are the essential boundary conditions written in (10) because the walls are electrically insulating. However, the Hartmann walls in this case are suposed to be perfectly conducting, which changes the boundary conditions to be imposed. Therefore, the boundary conditions imposed in a perfectly conducting wall are the natural conditions,

$$
\mathbf{b} \cdot \mathbf{n}=\mathbf{b}_{\text {ext }} \cdot \mathbf{n} \quad \text { and } \quad \mathbf{n} \times(\nabla \times \mathbf{b})=\mathbf{0} .
$$

The computation of the pressure gradient that drives the flow differs slightly from Shercliff's case,

$$
\frac{\mathrm{d} p}{\mathrm{~d} z}=\frac{K L^{3}}{\rho \nu^{2} \mathrm{Re}}, \quad \text { where } \quad K=\frac{\mathrm{Ha}}{1-0.95598 \mathrm{Ha}^{-1 / 2}-\mathrm{Ha}^{-1}} .
$$

The Hartmann number Ha corresponds to the norm of the external magnetic field because all the physical properties of the problem have been set to one. The proposed stabilized finite element method has been used to solve Hunt's problem for two different Hartmann number, Ha = 10 and $\mathrm{Ha}=100$. In both cases, the Reynolds number has been set to $\mathrm{Re}=10$. Again, each case has been solved with several meshes in order to be able to compare the numerical behavior of the method with regard to its theoretical convergence properties.

On one hand, the case with $\mathrm{Ha}=10$ and $\mathrm{Re}=10$ has been computed on a series of uniformly structured meshes of linear tetrahedral elements. Table 12 shows the same error norms as in the previous test, obtained for every mesh size $h$. The rate of convergence between two consecutive meshes is shown in brackets. These results have been displayed in Figure 8(a). It shows that the results obtained are in good agreement with the theory.

On the other hand, Hunt's problem with $\mathrm{Ha}=100$ and $\mathrm{Re}=10$ has been solved on a collection of structured meshes of linear tetrahedral elements. However, the meshes in this case are not uniformly structured, but with element concentration near the walls, because the boundary layers are much 


\begin{tabular}{|c|c|c|c|c|}
\hline$h$ & $\left\|\mathbf{e}_{\mathrm{u}}\right\|$ & $\left\|\nabla \mathbf{e}_{\mathrm{u}}\right\|$ & $\left\|\mathbf{e}_{\mathrm{b}}\right\|$ & $\left\|\nabla \times \mathbf{e}_{\mathrm{b}}\right\|$ \\
\hline \hline $2^{-2}$ & $8.98 \cdot 10^{-4}(-)$ & $1.63 \cdot 10^{-2}(-)$ & $5.25 \cdot 10^{-4}(-)$ & $9.97 \cdot 10^{-3}(-)$ \\
$2^{-3}$ & $2.55 \cdot 10^{-4}(1.82)$ & $8.53 \cdot 10^{-3}(0.93)$ & $1.38 \cdot 10^{-4}(1.93)$ & $4.84 \cdot 10^{-3}(1.04)$ \\
$2^{-4}$ & $6.92 \cdot 10^{-5}(1.88)$ & $4.08 \cdot 10^{-3}(1.06)$ & $4.21 \cdot 10^{-5}(1.71)$ & $2.42 \cdot 10^{-3}(1.00)$ \\
$2^{-5}$ & $1.87 \cdot 10^{-5}(1.89)$ & $1.81 \cdot 10^{-3}(1.17)$ & $1.40 \cdot 10^{-5}(1.59)$ & $1.21 \cdot 10^{-3}(1.00)$ \\
\hline
\end{tabular}

Table 12: Numerical errors and rate of convergence in brackets. Hunt's case with $\mathrm{Ha}=10$.

\begin{tabular}{|c|c|c|c|c|}
\hline$h^{*}$ & $\left\|\mathbf{e}_{\mathrm{u}}\right\|$ & $\left\|\nabla \mathbf{e}_{\mathrm{u}}\right\|$ & $\left\|\mathbf{e}_{\mathrm{b}}\right\|$ & $\left\|\nabla \times \mathbf{e}_{\mathrm{b}}\right\|$ \\
\hline \hline $2^{-2}$ & $1.34 \cdot 10^{-3}(-)$ & $2.82 \cdot 10^{-2}(-)$ & $1.19 \cdot 10^{-3}(-)$ & $2.33 \cdot 10^{-2}(-)$ \\
$2^{-3}$ & $1.54 \cdot 10^{-4}(3.12)$ & $8.62 \cdot 10^{-3}(1.71)$ & $1.16 \cdot 10^{-4}(3.36)$ & $6.09 \cdot 10^{-3}(1.94)$ \\
$2^{-4}$ & $1.17 \cdot 10^{-5}(3.72)$ & $1.18 \cdot 10^{-3}(2.87)$ & $1.32 \cdot 10^{-5}(3.14)$ & $1.41 \cdot 10^{-3}(2.11)$ \\
$2^{-5}$ & $2.13 \cdot 10^{-6}(2.46)$ & $8.61 \cdot 10^{-5}(3.78)$ & $1.86 \cdot 10^{-6}(2.83)$ & $1.52 \cdot 10^{-4}(3.21)$ \\
\hline
\end{tabular}

Table 13: Numerical errors and rate of convergence in brackets. Hunt's case with $\mathrm{Ha}=100$.

thinner than in the previous case of $\mathrm{Ha}=10$. Table 13 shows the computed error norms for the same quantities that in the case of $\mathrm{Ha}=10$. Again, the mesh-size $h^{*}$ corresponds to the equivalent mesh-size $h$ of a uniformly structured mesh with the same number of degrees of freedom. In Figure 8(b), the numerical results have been plotted. The same conclusion as in the previous results applies here, that is, the numerical behavior of the proposed method is very good in terms of convergence.

\subsection{Clogging of nozzles in steel casting processes.}

This numerical example serves two objectives. First, we aim at solving a convection-dominated problem to check that the designed formulation has the expected behavior in this regime and second, we want to solve a real problem with technological applications. The chosen problem is the clogging of nozzles in continuous casting of steel; see $[34,17]$ for a more detailed description. In continuous steel casting processes, the metal flow from the tundish to the mould is basically controlled by the tundish nozzle. Under particular flow conditions, there appears a serious problem which is the clogging of the nozzles due to the build-up of oxides. These build-ups can even block the flow through the nozzle. One of the solutions to this problem is the use of electromagnetic forces that reduce substantially the stagnation zone.

The L-shaped computational domain has been set as $[0,0.00795] \times[0,0.075] \cup[0,0.200] \times[0.075,0.525]$ due to the axial simmetry of the problem. This domain has been meshed with 21305 linear triangular elements and 10981 nodes, concentrating them in the nozzle zone. The hydrodynamic boundary conditions are a fixed velocity $\mathbf{u}=(0,-0.05)$ at the inlet $\{y=0.525\}$, a free surface condition at the outlet $\{y=0\}$, symmetric conditions $u_{x}=0$ at the symmetry axes $\{x=0\}$ and no-slip conditions $\mathbf{u}=(0,0)$ elsewhere. The boundary conditions for the magnetic unknowns have been set as symmetric conditions $b_{x}=0$ at $\{x=0\}$, and a fixed radial magnetic induction field $b_{x}=b_{\text {ext }, x}$ elsewhere. Finally, the gravity $\mathbf{g}=(0,-9.81)$ is set as an external body force. 


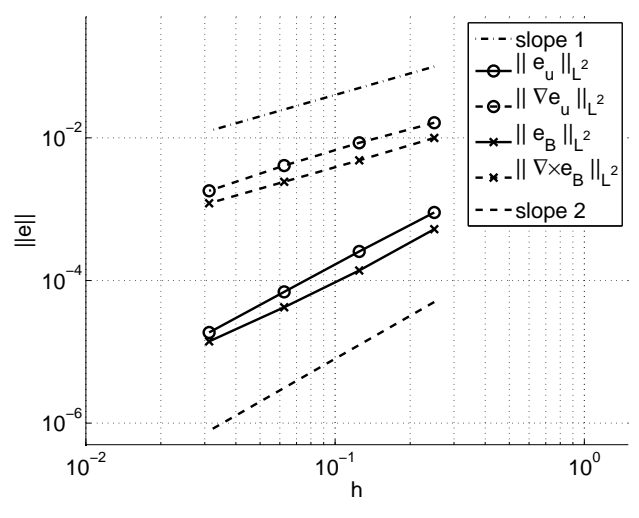

(a) $\mathrm{Ha}=10$

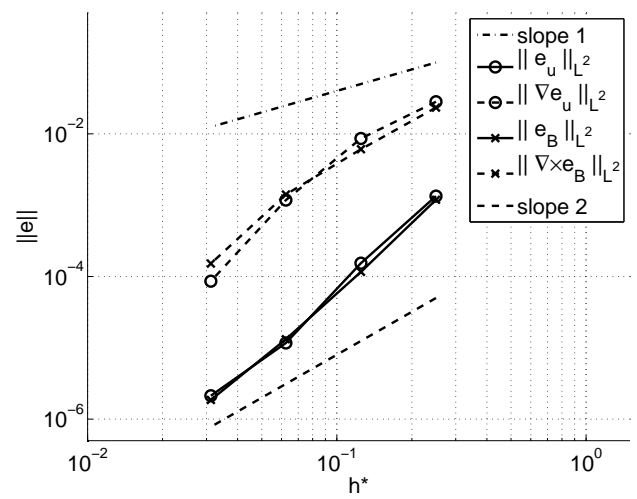

(b) $\mathrm{Ha}=100$

Figure 8: Convergence plots of velocity and induction error norms for Hunt's case.

The physical properties of the problem have been taken as $\rho=7 \times 10^{3}, \nu=2 \times 10^{-6}, \sigma=10^{6}$ and $\mu_{\mathrm{m}}=8 \times 10^{-7}$. The flow parameters and the nozzle length lead to a Reynolds number of $\mathrm{Re} \approx 20000$. The problem has been solved with two Hartmann numbers $(\mathrm{Ha}=1,100)$ to study the flow behavior under weak and strong external magnetic fields.

The obtained results are shown in Figure 9, where the velocity field streamlines in the nozzle zone are plotted. Figure 9(a) shows the case with $\mathrm{Ha}=1$ where it is clearly seen that the flow separates from the internal wall of the nozzle generating a stagnation zone which produces the clogging. However, increasing the external magnetic field reduces drastically this effect. Figure 9(b) shows the velocity streamlines for $\mathrm{Ha}=100$. In this case, the flow becomes attached in the nozzle zone, reducing the recirculation zones and therefore eliminating the clogging of the nozzles.

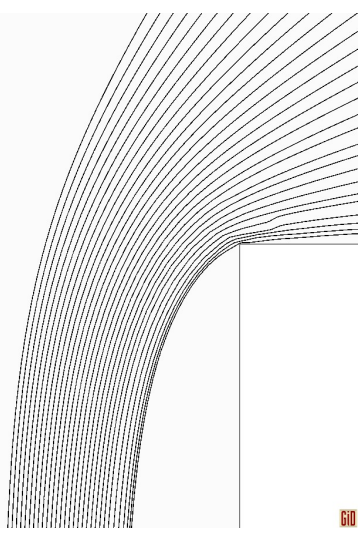

(a) $\mathrm{Ha}=1$

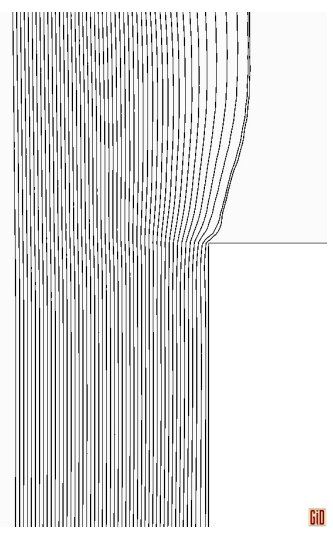

(b) $\mathrm{Ha}=100$

Figure 9: Velocity streamlines in the nozzle zone. 


\section{Conclusions}

A finite element approximation of the resistive MHD problem has been proposed in this work. The formulation falls within the category of stabilized finite element methods and, as such, is intended to avoid the need for using finite element approximations satisfying the compatibility conditions of the continuous problem and dealing with ranges of the physical parameters in which first order derivatives dominate second order ones.

Particular features of the formulation proposed are that it is of residual type, can be based on the VMS framework and the stabilization parameters are designed from the numerical analysis in [3, 5], accounting for the coupling between the fluid and magnetic sub-problems. However, the most salient feature is that it allows to converge to singular solutions even when using a continuous Lagrangian approximation for the magnetic induction field. To our knowledge, this is the first time this is achieved. From the technical point of view, this possibility relies on the splitting of the residual of the finite element equation for the magnetic induction into two parts, one consisting of the gradient of the magnetic pseudo-pressure and the other the remaining terms. This is possible because the magnetic pseudo-pressure is zero for the continuous problem. The two resulting residuals are multiplied by stabilization parameters with different asymptotic behavior, a key point to mimic the correct functional setting of the continuous problem at the discrete level.

Support to assess the feasibility of our formulation arises from two facts. On the one hand, its applicability to the full transient and nonlinear problem has been demonstrated in a set of numerical experiments which have shown its excellent performance. On the other hand, we have performed a complete numerical analysis of the linearized and stationary MHD problem in [5]. In particular, optimal stability and convergence results have been obtained.

\section{Acknowledgements}

The work of the first author was funded by the European Research Council under the FP7 Programme Ideas through the Starting Grant No. 258443 - COMFUS: Computational Methods for Fusion Technology. The second author has been partially supported by the Consolider-Ingenio project TECNO-

FUS, Ref. CSD2008-00079, from the Spanish Ministry of Science and Innovation. Finally, the third author would like to acknowledge the support received from the Universitat Politècnica de Catalunya (UPC) and from the Col.legi d'Enginyers de Camins, Canals i Ports de Catalunya.

\section{References}

[1] F. Armero and J. C. Simo. Long-term dissipativity of time-stepping algorithms for an abstract evolution equation with applications to the incompressible MHD and Navier-Stokes equations. Computer Methods in Applied Mechanics and Engineering, 131(1-2):41 - 90, 1996.

[2] S. Badia and R. Codina. A combined nodal continuous-discontinuous finite element formulation for the Maxwell problem. Applied Mathematics and Computation, 218(8):4276-4294, 2011. 
[3] S. Badia and R. Codina. A nodal-based finite element approximation of the Maxwell problem suitable for singular solutions. SIAM Journal on Numerical Analysis, 50(2):398-417, 2012.

[4] S. Badia and R. Codina. Stokes, Maxwell and Darcy: A single finite element approximation for three model problems. Applied Numerical Mathematics, 62(4):246-263, 2012.

[5] S. Badia, R. Codina, and Planas R. Analysis of a unconditionally convergent stabilized finite element formulation for incompressible magnetohydrodynamics. In preparation, 2012.

[6] N. Ben Salah, A. Soulaimani, and W.G. Habashi. A finite element method for magnetohydrodynamics. Computer Methods in Applied Mechanics and Engineering, 190(43-44):5867 - 5892, 2001.

[7] N. Ben Salah, A. Soulaimani, W.G. Habashi, and M. Fortin. A conservative stabilized finite element method for the magneto-hydrodynamic equations. International Journal for Numerical Methods in Fluids, 29(5):535-554, 1999.

[8] F. Brezzi and M. Fortin. Mixed and Hybrid Finite Element Methods. Springer Verlag, 1991.

[9] A.N. Brooks and T.J.R. Hughes. Streamline upwind / Petrov-Galerkin formulations for convection dominated flows with particular emphasis on the incompressible Navier-Stokes equation. Computer Methods in Applied Mechanics and Engineering, 32:199-259, 1982.

[10] A. Buffa, P. Ciarlet Jr., and E. Jamelot. Solving electromagnetic eigenvalue problems in polyhedral domains with nodal finite elements. Numerische Mathematik, 113(4):497-518, 2009.

[11] L. Chacón. An optimal, parallel, fully implicit Newton-Krylov solver for three-dimensional viscoresistive magnetohydrodynamics. Physics of Plasmas, 15(5):056103, 2008.

[12] L. Chacón. Scalable parallel implicit solvers for 3D magnetohydrodynamics. J. Phys.: Conf. Ser., 125:012041, 2008.

[13] R. Codina. Comparison of some finite element methods for solving the diffusion-convectionreaction equation. Computer Methods in Applied Mechanics and Engineering, 156:185-210, 1998.

[14] R. Codina. Stabilized finite element approximation of transient incompressible flows using orthogonal subscales. Computer Methods in Applied Mechanics and Engineering, 191:42954321, 2002.

[15] R. Codina. Analysis of a stabilized finite element approximation of the Oseen equations using orthogonal subscales. Applied Numerical Mathematics, 58:264-283, 2008.

[16] R. Codina and N. Hernández. Stabilized finite element approximation of the stationary MHD equations. Computational Mechanics, 38:344-355, 2006.

[17] R. Codina and N. Hernández. Approximation of the thermally coupled MHD problem using a stabilized finite element method. Journal of Computational Physics, 230(4):1281 - 1303, 2011.

[18] R. Codina, J. Principe, O. Guasch, and S. Badia. Time dependent subscales in the stabilized finite element approximation of incompressible flow problems. Computer Methods in Applied Mechanics and Engineering, 196:2413-2430, 2007. 
[19] M. Costabel. A coercive bilinear form for Maxwell's equations. Journal of Mathematical Analysis and Applications, 157(2):527-541, 1991.

[20] M. Costabel and M. Dauge. Singularities of electromagnetic fields in polyhedral domains. Archives for Rational Mechanics and Analysis, 151(3):221-276, 2000.

[21] M. Costabel and M. Dauge. Weighted regularization of Maxwell equations in polyhedral domains. Numerische Mathematik, 93(2):239-277, 2002.

[22] P.A. Davidson. An introduction to magnetohydrodynamics. Cambridge University Press, 2001.

[23] A. Ern and J.L. Guermond. Theory and Practice of Finite Elements. Springer-Verlag, 2004.

[24] J.-F. Gerbeau. A stabilized finite element method for the incompressible magnetohydrodynamic equations. Numerische Mathematik, 87:83-111, 2000.

[25] J.F. Gerbeau, C. Le Bris, and T. Lelièvre. Mathematical methods for the magnetohydrodynamics of liquid metals. Oxford University Press, USA, 2006.

[26] M.D. Gunzburger, A.J. Meir, and J.P. Peterson. On the existence, uniqueness, and finite element approximation of solutions of the equations of stationary, incompressible magnetohydrodynamics. Math. Comp., 56:523-563, 1991.

[27] U. Hasler, A. Schneebeli, and D. Schötzau. Mixed finite element approximation of incompressible MHD problems based on weighted regularization. Applied Numerical Mathematics, 51:19 $-45,2004$.

[28] C. Hazard and M. Lenoir. On the solution of time-harmonic scattering problems for Maxwell's equations. SIAM Journal on Mathematical Analysis, 27(6):1597-1630, 1996.

[29] P. Houston, D. Schötzau, and X. Wei. A mixed DG method for linearized incompressible magnetohydrodynamics. Journal of Scientific Computing, 40:281-314, 2009.

[30] T.J.R. Hughes. Multiscale phenomena: Green's function, the Dirichlet-to-Neumann formulation, subgrid scale models, bubbles and the origins of stabilized formulations. Computer Methods in Applied Mechanics and Engineering, 127:387-401, 1995.

[31] T.J.R. Hughes, G.R. Feijóo, L. Mazzei, and J.B. Quincy. The variational multiscale method-a paradigm for computational mechanics. Computer Methods in Applied Mechanics and Engineering, 166:3-24, 1998.

[32] J.C.R. Hunt. Magnetohydrodynamic flow in rectangular ducts. Journal of Fluid Mechanics, 21:577-590, 1965.

[33] A. Kritz and D. Keyes. Fusion simulation project workshop report. Journal of Fusion Energy, 28:1-59, 2009.

[34] J.D. Lavers and L. Kadar. Application of electromagnetic forces to reduce tundish nozzle clogging. Applied Mathematical Modelling, 45:29-45, 2004.

[35] P. Monk. Finite Element Methods for Maxwell's Equations. Oxford University Press, 2003. 
[36] J. C. Nedelec. Mixed finite elements in $\mathbb{R}^{3}$. Numer. Meth., 35:119-136, 1980.

[37] J. C. Nedelec. A new family of mixed finite elements in $\mathbb{R}^{3}$. Numer. Meth., 50:57-81, 1986.

[38] R. Planas, S. Badia, and R. Codina. Approximation of the inductionless mhd problem using a stabilized finite element method. Journal of Computational Physics, 230(8):2977 - 2996, 2011.

[39] A. Prohl. Convergent finite element discretizations of the nonstationary incompressible magnetohydrodynamics system. ESAIM: Mathematical Modelling and Numerical Analysis, 42(06):1065-1087, 2008.

[40] D. Schötzau. Mixed finite element methods for stationary incompressible magnetohydrodynamics. Numerische Mathematik, 96:771-800, 2004.

[41] J.N. Shadid, R.P. Pawlowski, J.W. Banks, L. Chacon, P.T. Lin, and R.S. Tuminaro. Towards a scalable fully-implicit fully-coupled resistive MHD formulation with stabilized FE methods. Journal of Computational Physics, 229(20):7649 - 7671, 2010.

[42] J.A. Shercliff. Steady motion of conducting fluids in pipes under transverse magnetic fields. Proceedings of Cambridge Philosophical Society, 49:126-144, 1953.

[43] T. Sorokina and A.J. Worsey. A multivariate Powell-Sabin interpolant. Advances in Computational Mathematics, 29(1):71-89, 2008.

[44] R. Temam. Navier-Stokes equations. North-Holland, 1984.

[45] A. Toselli and O.B. Widlund. Domain decomposition methods-algorithms and theory. Springer Verlag, 2005. 